\title{
Aeroacoustic source mechanisms of a wavy leading edge undergoing vortical disturbances
}

\author{
Jacob M. Turner and Jae Wook Kim $\dagger$ \\ Aerodynamics \& Flight Mechanics Research Group, University of Southampton \\ Southampton, SO17 1BJ, United Kingdom \\ (Received xx; revised xx; accepted xx)
}

High-accuracy numerical simulations are performed to study aeroacoustic source mechanisms of wavy leading edges (WLE) on a thin aerofoil undergoing vortical disturbances. This canonical study is based on a prescribed spanwise vortex travelling downstream and creating secondary vortices as it passes through the aerofoil's leading edge. The primary aim of the study is to precisely understand the relationships between the vortexinduced velocity perturbation and the wall pressure fluctuation on the WLE geometry. It is observed that by increasing the size (amplitude) of the WLE the source strength at the peak region is reduced rapidly to a certain point and followed by a saturation stage, while at the root (trough) it remains fairly consistent regardless of the WLE size. This observation is demonstrated to be the consequence of three-dimensional vortex dynamics taking place along the WLE. One of the most profound features is that a system of horseshoe-like secondary vortices are created from the WLE peak region upon the impingement of the prescribed vortex. It is found that the horseshoe vortices produce significantly non-uniform velocity perturbation in front of the WLE leading to the disparity in the source characteristics between the peak and root. The alterations to the impinging velocity perturbation are carefully analysed and related to the wall pressure fluctuation in this study. In addition, a semi-analytic model based on Biot-Savart's law is developed to better understand and explain the role of the horseshoe vortex systems and the source mechanisms.

\section{Introduction}

When an upstream vortical field impinges on a solid body its normal velocity component is abruptly forced to zero due to a no-penetration condition. This results in the generation of pressure fluctuation on the surface, which in the case of an aerofoil radiate as a dipole sound primarily from the aerofoil leading edge (LE). Accordingly this noise mechanisms is usually referred to as LE noise or aerofoil-turbulence interaction (ATI) noise, and is often considered to be one of the major noise sources particularly in the presence of significant upstream disturbances (Migliore \& Oerlemans 2004). Such conditions are regularly met in a variety of engineering applications, for example contrarotating fans, propellers, turbofan outlet-guide vanes, high-lift devices and wind farms. Over the years many approaches have been adopted for the study of ATI noise to understand both the physical noise generation mechanisms and the influence of various geometric parameters such as aerofoil thickness, angle of attack and camber. This includes the theoretical model of Amiet (1975) and its subsequent extensions, namely, the convection of two-dimensional frozen turbulence in a uniform mean flow (Roger \&

$\dagger$ Email address for correspondence: j.w.kim@soton.ac.uk 
Moreau 2010); theoretical and numerical approaches based on harmonic gusts (Goldstein 1978; Atassi et al. 1990; Myers \& Kerschen 1995, 1997; Lockard \& Morris 1998; Evers \& Peake 2000; Gill et al. 2013; Ayton \& Peake 2013, 2015); experimental approaches based on grid generated homogeneous isotropic turbulence (Paterson \& Amiet 1976; Moreau et al. 2005; Devenport et al. 2010); and, recent computational approaches based on twoor three-dimensional Euler and Navier-Stokes simulations (Christophe et al. 2007, 2008; Deniau et al. 2011; Gill et al. 2015; Kim \& Haeri 2015).

Research conducted in the previous a few years has shown that a potential passive treatment capable of large reductions to ATI noise is the inclusion of an undulated (wavy) aerofoil leading edge. Such geometry was originally considered for its aerodynamic properties (Miklosovic et al. 2004; Johari et al. 2007; Hansen et al. 2011, 2016), and is inspired by the leading-edge protrusions (tubercles) of the humpback whale's pectoral flippers thought to be responsible for its impressive maneuverability when catching its prey (Fish et al. 2008). One of the first attempts to quantify the aeroacoustic properties of WLEs was conducted by Hansen et al. (2012), who through experiments based on a NACA0021 aerofoil identified that WLE cases obtained large tonal and broadband self-noise reductions within a certain frequency range. They theorised that the noise reductions were related to the production of streamwise vortices at the leading-edge troughs, which enhance the stability of the boundary layer by mixing in higher momentum fluid from the free stream altering the generation of vorticity near the trailing edge. In the meantime, a large effort has been made towards quantifying the extent of ATI noise reductions based on various geometric parameters, particularly the WLE amplitude $\left(h_{L E}\right)$ and wavelength $\left(\lambda_{L E}\right)$ (Lau et al. 2013; Clair et al. 2013; Narayanan et al. 2015; Chaitanya et al. 2015; Kim et al. 2016). It is consistently found that the level of noise reductions is positively influenced by increasing $h_{L E}$. Comparatively, altering $\lambda_{L E}$ seems to have a smaller effect.

Narayanan et al. (2015) performed an experimental parametric study based on gridgenerated homogeneous isotropic turbulence and noted that more noise reductions were achievable for lower flow speeds $\left(U_{\infty}\right)$ and thinner aerofoils. One of their most significant findings was a consistent starting frequency of noise reduction: $f_{0}=\alpha U_{\infty} /\left(2 h_{L E}\right)$, where $\alpha \approx 0.5$ for all cases. This finding is also supported by the work of Clair et al. (2013) who observed a broader frequency range of noise reductions for a reduced flow speed. A further parametric study was conducted by Chaitanya et al. (2015) focusing on the WLE serration angle defined as $\theta=\tan ^{-1}\left(4 h_{L E} / \lambda_{L E}\right)$. They concluded that, for a given WLE amplitude and turbulence integral length scale, there exists an optimum serration angle for maximum noise reduction. This means that increasing $\lambda_{L E}$ can result in either an increase of the noise reduction or a decrease depending on whether $\theta$ is higher than the optimal value or lower, which explains some inconsistent observations (on the effect of $\lambda_{L E}$ ) made between Lau et al. (2013) and Narayanan et al. (2015). Chaitanya et al. (2015) also showed that the noise reduction increases with frequency as a power law. Meanwhile, an analytical study by Mathews \& Peake (2015) suggested that there exist noise-increasing eddy components in the impinging turbulence depending on their orientation relative to the leading edge. It is, however, speculated that the probability of such eddies is lower in the WLE cases and the averaged effect yields a consistent noise reduction.

Despite rapid growth in the field, understandings of the noise reduction mechanisms associated with WLEs are still underdeveloped. A significant progress has recently been made by Kim et al. (2016) who carried out high fidelity numerical simulations based on three-dimensional nonlinear compressible Euler equations and synthetic inflow turbulence (Kim \& Haeri 2015). They identified two primary noise reduction mechanisms: a source 
cutoff effect and a phase interference effect. The source cutoff effect was recognised by analysing the auto-spectrum of the fluctuating wall pressure at different points along the WLE, revealing a substantial reduction in source strength at the hill region across the whole frequency range. It was suggested that this was due to the geometric obliqueness relative to the incoming flow turbulence. Arguably more intriguing is that significant source strength differences were observed between the peak and root, despite the fact that both has zero obliqueness against the incoming disturbances similar to a straight leading edge (SLE) case. At low frequencies the peak exhibited a substantially lower source strength than the root, while at high frequencies the peak seemed to overtake the root by a small margin. Overall, the source strength at the root was comparable to that of the SLE at all frequencies. Regarding the phase interference effect, it was reported that the two-point phase spectra between the peak and other points along the WLE experiences an increased level of phase shifts towards $\pi$ (out of phase) compared to the SLE case.

Guided by the earlier findings, the present work is aimed to extend the understanding of the aeroacoustic source mechanisms associated with the WLEs interacting with vortical disturbances. Here we focus on explaining the disparity in source characteristics between the peak and root as outlined above. The current study is based on a computational approach similar to Kim \& Haeri (2015); Kim et al. (2016) except that a single spanwise vortex model is used instead of synthetic inflow turbulence to create vortical disturbances. Using the single vortex model has two main advantages over the synthetic turbulence approach. First, it offers enhanced clarity in observing and identifying major physical phenomena taking place during the aerofoil-vortex interaction process. Secondly, there still is a reasonable range of frequency components in the vortical disturbances unlike harmonic gust approaches and the resulting spectra are much cleaner than those from the synthetic turbulence approach.

The current simulation result shows that the prescribed vortex impinging on the aerofoil creates secondary vortices along the WLE which form a horseshoe-like vortex system which tends to intensify as time elapses. The three-dimensional dynamics of the horseshoe vortex system is carefully investigated with regard to the induced velocity field around the WLE, from which the wall pressure fluctuation (aeroacoustic source strength) is deduced. The problem is also modelled semi-analytically by using BiotSavart's law to further support the hypothesis proposed in the current study. It should be noted that the current study is still based on a zero-thickness aerofoil in an inviscid flow at zero mean incidence angle and low Mach number. It is expected that a realistic aerofoil (with a thickness and/or camber) and a non-uniform viscous mean flow may develop increasingly complex mechanisms associated with self-generated leading-edge vortices (Hansen et al. 2016) and viscous distortion/dissipation of the vortices. Prior to the additional complexity to be investigated, the current study focuses on the most basic form of the aerofoil-vortex interaction and therefore the simplest geometry and flow condition are chosen in this paper.

This paper is structured and written in the following order. $\S 2$ introduces the computational set-up and methods used in this study including details of the prescribed spanwise vortex model implemented in the current simulations. $\S 3$ provides initial observations obtained from the simulations including wall and far-field pressure spectra for various WLE geometries. Additionally it includes a qualitative discussion of the secondary vortex structures created along the WLE and how they influence the induced velocity field. $§ 4$ provides quantitative explanations as to how the aeroacoustic source strength at the root region is maintained at a level similar to the SLE case. $\S 5$ moves the focus on to the peak region and explain the trend of the source strength decaying (but converging) with the 

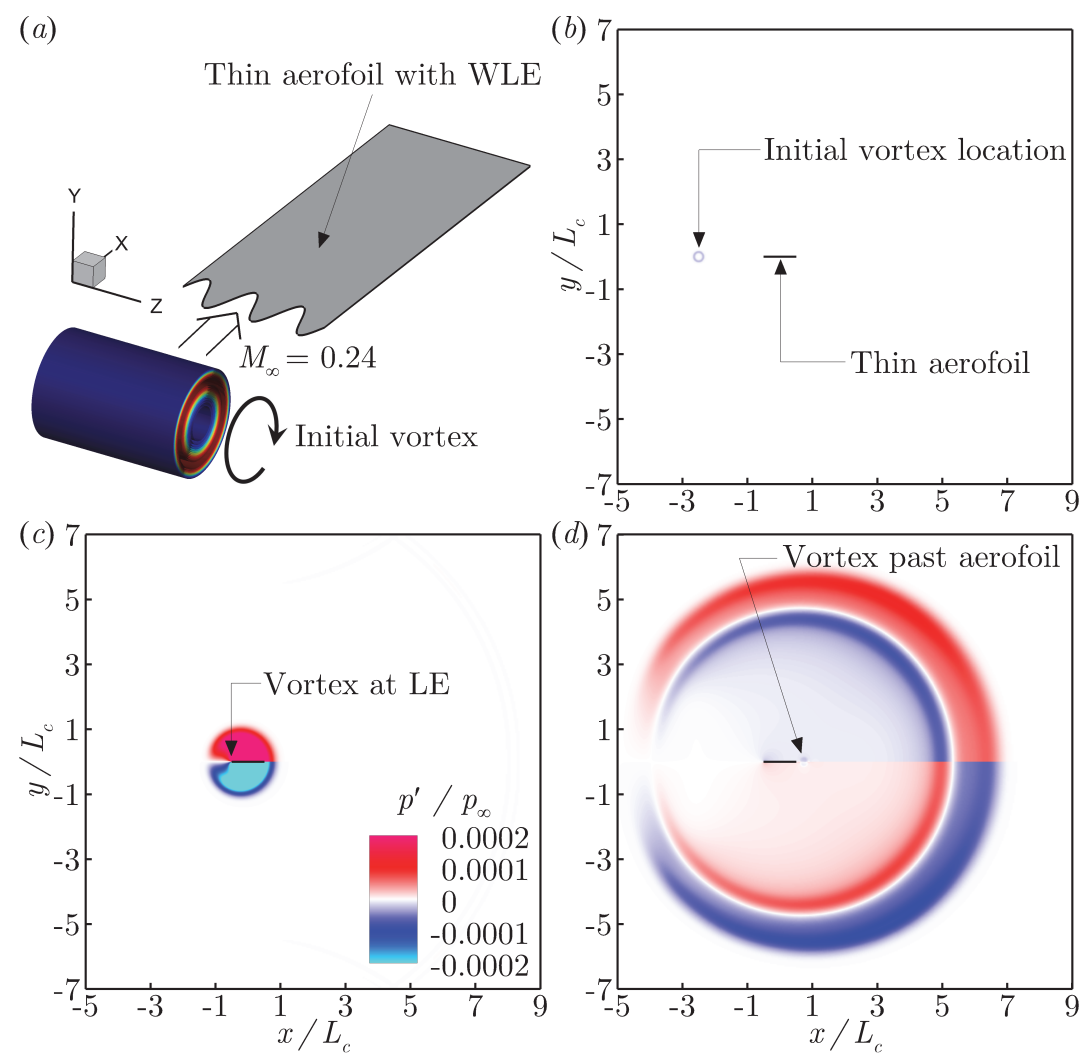

FiguRE 1. Description of the current problem: a prescribed spanwise vortex impinging on a thin aerofoil with a wavy leading edge: $(a)$ schematic diagram, $(b)$ initial condition, $(c)$ during the interaction and $(d)$ after the interaction. The mean aerofoil chord is denoted by $L_{c}$.

WLE amplitude, which is also supported by the semi-analytic model derived from BiotSavart's law. In $\S 6$ supplementary observations are made with respect to a similarity rule in the source strength and the effect of three-dimensionality in the impinging vortex. Finally concluding remarks are provided in $\S 7$.

\section{Description of the problem and computational set-up}

The current problem of aerofoil noise generation due to interaction with a prescribed spanwise vortex impinging on the leading edge is illustrated in figure 1. Instantaneous snapshots of the computed pressure field exhibit sound waves generated and radiated during and after the interaction. This section describes the problem set-up and the computational methodologies used in this study.

\subsection{Computational domain and aerofoil geometry}

The computational domain in a rectangular cuboid contains a flat-plate aerofoil at the centre with zero thickness and zero angle of attack. The zero-thickness aerofoil is modelled by using a H-topology grid system where the central branch section represents the aerofoil's upper and lower surfaces with no gap between them. The longitudinal and vertical boundaries of the domain are surrounded by a sponge layer through which the flow is (gently) forced to maintain the potential mean flow condition. Any acoustic 


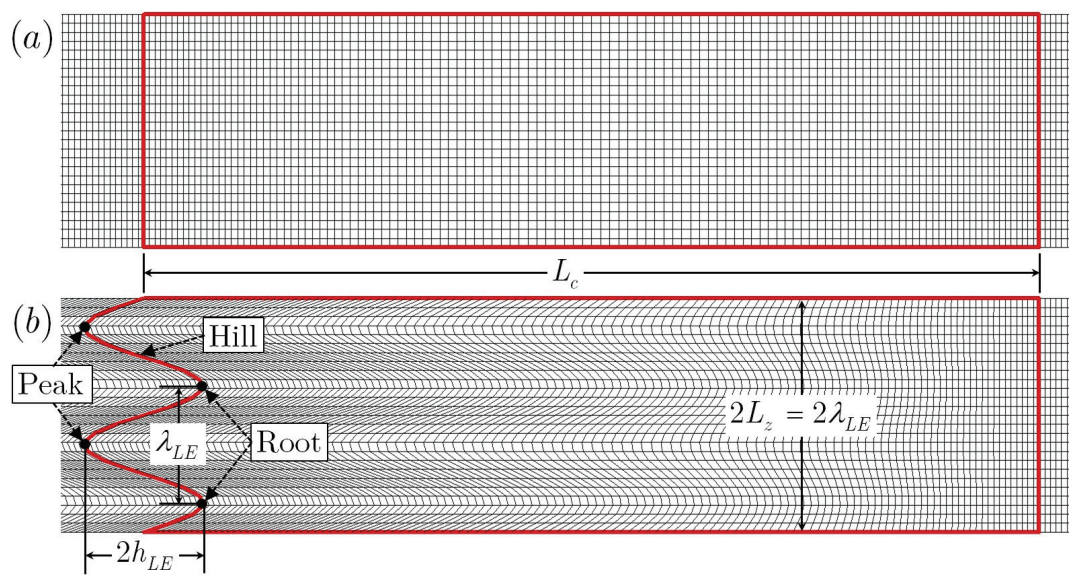

FiguRE 2. Surface meshes (coarsened for illustration purposes) on the flat-plate aerofoils used in the present study, with $(a)$ straight and $(b)$ wavy leading edges (SLE and WLE, respectively). The case of $h_{L E} / L_{c}=1 / 15$ and $\lambda_{L E} / L_{c}=2 / 15$ is shown in this figure.

waves are attenuated and absorbed in the sponge layer to prevent numerical reflections at the outer boundaries. The lateral boundaries of the domain are interconnected via a periodic boundary condition. The entire computational domain; the inner region (physical domain) where meaningful simulation data are obtained; and, the sponge layer zone are defined as

$$
\left.\begin{array}{c}
\mathcal{D}_{\infty}=\left\{\boldsymbol{x} \mid x / L_{c} \in[-5,9], y / L_{c} \in[-7,7], z \in\left[-\frac{1}{2} L_{z}, \frac{1}{2} L_{z}\right]\right\}, \\
\mathcal{D}_{\text {physical }}=\left\{\boldsymbol{x} \mid x / L_{c} \in[-3,3], y / L_{c} \in[-5,5], z \in\left[-\frac{1}{2} L_{z}, \frac{1}{2} L_{z}\right]\right\}, \\
\mathcal{D}_{\text {sponge }}=\mathcal{D}_{\infty}-\mathcal{D}_{\text {physical }},
\end{array}\right\}
$$

where $L_{c}$ denotes the mean chord length of the aerofoil and $L_{z}$ is the spanwise length of the domain. The origin of the domain is located at the centre of the aerofoil surface. In the current simulations, $L_{z}$ is set to cover one wavelength of the WLE profile given. The free-stream Mach number is set to $M_{\infty}=u_{\infty} / a_{\infty}=0.24$. This Mach number is the same as used in the previous work by Kim et al. (2016).

The aerofoil has a WLE (wavy leading edge) which is profiled by using a sine function where the most protruded points are defined as "peak", the least as "root" and the middle as "hill" as denoted in figure 2 . Herein, $h_{L E}$ is the WLE amplitude $\left(2 h_{L E}\right.$ being the peak-to-root amplitude) and $\lambda_{L E}$ is the spanwise wavelength of the WLE. The WLE profile in this study is defined by

$$
x_{L E}(z)=-\frac{1}{2} L_{c}+h_{L E} \sin \left(\frac{2 \pi z}{\lambda_{L E}}\right), \quad z \in\left[-\frac{1}{2} L_{z}, \frac{1}{2} L_{z}\right],
$$

where the location of the trailing edge is fixed at $x_{T E}=\frac{1}{2} L_{c}$. The spanwise coordinates of the peak, hill centre and root are $z=-\lambda_{L E} / 4,0$ and $\lambda_{L E} / 4$, respectively. The default wavelength of the WLE in the current study is $\lambda_{L E} / L_{c}=2 / 15$ and in $\S 6 \lambda_{L E} / L_{c}=4 / 15$ is used as well. The baseline WLE amplitude is $h_{L E} / L_{c}=1 / 15$ and lower/higher values of $h_{L E}$ are also explored to investigate the effect of $h_{L E}$. These geometric parameters are similar to those used in Kim et al. (2016). 


\subsection{Governing equations and numerical methods}

Aerofoil noise generated by interaction with vortical disturbances is largely considered an inviscid phenomenon and therefore exclusion of viscous terms is usually considered to be a reasonable simplification (Goldstein 1978; Atassi et al. 1990; Myers \& Kerschen 1995; Lockard \& Morris 1998; Evers \& Peake 2000; Ayton \& Peake 2013; Gill et al. 2015; Kim \& Haeri 2015). Following up on the historical approach, we employ full 3-D compressible Euler equations (with a source term for the sponge layer mentioned earlier) in a conservative form transformed onto a generalised coordinate system:

$$
\frac{\partial}{\partial t}\left(\frac{\boldsymbol{Q}}{J}\right)+\frac{\partial}{\partial \xi_{i}}\left(\frac{\boldsymbol{F}_{j}}{J} \frac{\partial \xi_{i}}{\partial x_{j}}\right)=-\frac{a_{\infty}}{L_{c}} \frac{\boldsymbol{S}}{J}
$$

where $i=1,2,3$; and, $j=1,2,3$ denoting the three dimensions. In (2.3), the conservative variable and flux vectors are given by

$$
\left.\begin{array}{c}
\boldsymbol{Q}=\left[\rho, \rho u, \rho v, \rho w, \rho e_{\mathrm{t}}\right]^{T}, \\
\boldsymbol{F}_{j}=\left[\rho u_{j},\left(\rho u u_{j}+\delta_{1 j} p\right),\left(\rho v u_{j}+\delta_{2 j} p\right),\left(\rho w u_{j}+\delta_{3 j} p\right),\left(\rho e_{\mathrm{t}}+p\right) u_{j}\right]^{T},
\end{array}\right\}
$$

where $\xi_{i}=\{\xi, \eta, \zeta\}$ are the generalised coordinates, $x_{j}=\{x, y, z\}$ are the Cartesian coordinates, $u_{j}=\{u, v, w\}, e_{\mathrm{t}}=p /[(\gamma-1) \rho]+u_{j} u_{j} / 2$ and $\gamma=1.4$ for air. In the current setup, $\xi, \eta$ and $\zeta$ are body fitted coordinates along the grid lines in the streamwise, vertical and lateral directions, respectively. The Jacobian determinant of the coordinate transformation (from Cartesian to the body fitted) is given by $J^{-1}=|\partial(x, y, z) / \partial(\xi, \eta, \zeta)|$ (Kim \& Morris 2002). The extra source term $\boldsymbol{S}$ on the right-hand side of (2.3) is non-zero within the sponge layer only, which is described in Kim et al. (2010).

In this work, the governing equations given above are solved by using high-order accurate numerical methods specifically developed for aeroacoustic simulations on structured grids. The flux derivatives in space are calculated based on fourth-order pentadiagonal compact finite difference schemes with seven-point stencils (Kim 2007). Explicit time advancing of the numerical solution is carried out by using the classical fourth-order Runge-Kutta scheme with the CFL number of 0.95 . Numerical stability is maintained by implementing sixth-order pentadiagonal compact filters for which the cutoff wavenumber (normalised by the grid spacing) is set to $0.85 \pi$ (Kim 2010). In addition to the sponge layers used, characteristics-based non-reflecting boundary conditions (Kim \& Lee 2000) are applied at the far-boundaries in order to prevent any outgoing waves from returning to the computational domain. Periodic conditions are used across the spanwise boundary planes as indicated earlier. Slip wall (no penetration) boundary conditions are implemented on the aerofoil surface (Kim \& Lee 2004) and also on the centre plane downstream of the aerofoil. The latter is intended to eliminate the secondary interaction which takes place at the trailing edge, and therefore to focus on the leading edge interaction only.

The simulation is carried out on a total of $43,760,640$ grid cells $(1036 \times 660 \times 64)$ where the smallest cells are located at the aerofoil LE with the size of $\Delta x=\Delta y=0.00625 L_{c}$ and $\Delta z=0.002083 L_{c}$. Although the grid is gradually stretched outwards, a high grid resolution is still maintained in the far field in order to capture high-frequency components radiated. At least 46 cells are located across the diameter of the initial vortex. The computation is parallelised via domain decomposition and message passing interface (MPI) approaches. The compact finite difference schemes and filters used are implicit in space due to the inversion of pentadiagonal matrices involved, which requires a precise and efficient technique for the parallelisation in order to avoid numerical artefacts that may appear at the subdomain boundaries. A recent parallelisation approach based on quasi-disjoint matrix systems (Kim 2013) offering super-linear scalability is used in 

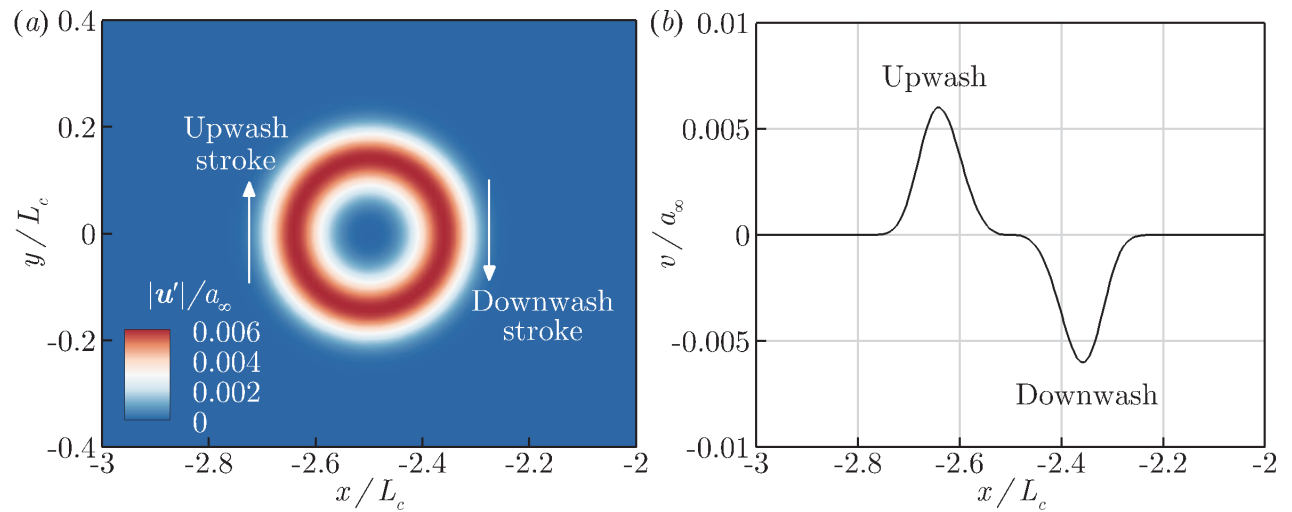

Figure 3 . The initial velocity field induced by the spanwise vortex model given by $(2.6):(a)$ induced velocity magnitude contours and $(b)$ the vertical velocity profile along the centreline $(y=0)$.

the present paper. The entire domain is decomposed and distributed onto 480 separate computing nodes/subdomains $(20 \times 12 \times 2$ in the streamwise, vertical and spanwise directions, respectively). A grid convergence test has been performed for the current computational set-up - see Appendix A.

\subsection{Prescribed spanwise vortex model}

The spanwise (two-dimensional) vortex model prescribed as an initial condition in this study is based on the Gaussian synthetic eddy profile used in previous publications (Kim \& Haeri 2015; Kim et al. 2016). It provides a divergence-free velocity field via taking the curl of the following vector potential, i.e. $\boldsymbol{u}^{\prime}(\boldsymbol{x}, t=0)=\nabla \times\left[\Psi(\boldsymbol{x}) \boldsymbol{e}_{z}\right]$ :

$$
\Psi(\boldsymbol{x})=a_{\infty} L_{c} \frac{\epsilon}{\sqrt{\sigma}} \exp \left\{-[3 \sigma r(\boldsymbol{x})]^{2}\right\}, \quad r(\boldsymbol{x})=\frac{\left(x-x_{0}\right)^{2}+y^{2}}{L_{c}^{2}},
$$

where $\epsilon$ and $\sigma$ are control parameters for the vortex strength and size, respectively; $x_{0}=-2.5 L_{c}$ is the streamwise coordinate of the starting point of the vortex core; and, $\boldsymbol{e}_{z}$ is a Cartesian unit vector in the spanwise direction. This results in the following formulae for the velocity field at $t=0$ :

$$
u(\boldsymbol{x})=u_{\infty}+36 \frac{\sigma^{2}}{L_{c}^{2}} y r(\boldsymbol{x}) \Psi(\boldsymbol{x}), \quad v(\boldsymbol{x})=-36 \frac{\sigma^{2}}{L_{c}^{2}}\left(x-x_{0}\right) r(\boldsymbol{x}) \Psi(\boldsymbol{x}),
$$

while the pressure and the density are set to ambient/quiescent conditions $\left(p_{\infty}\right.$ and $\left.\rho_{\infty}\right)$. The control parameters are set to $\epsilon=0.00228$ and $\sigma=14.4$ such that the level and the length scale of the velocity perturbation $\left(0.025 u_{\infty}\right.$ and $0.1 L_{c}$, respectively) are similar to those of the large eddies used in the previous experiments by Narayanan et al. (2015) and Chaitanya et al. (2015). The parameters were determined via the following definition of perturbation length scale in this paper:

$$
\text { Perturbation-Length-Scale }=\frac{1}{\max |v(x)|} \int_{x_{0}}^{x_{0}+\infty}|v(x)| \mathrm{d} x \quad \text { at } \quad y=z=t=0 .
$$

The resulting velocity field is plotted in figure 3 . The prescribed vortex induces clockwise rotating velocity components, firstly making a downwash stroke on to the aerofoil leading edge followed by an upwash stroke as the vortex travels further downstream. It is shown in figure 1 that the downwash-upwash strokes create compression-expansion pressure 
waves on the upper side of the leading edge radiating towards the top (with the opposite taking place on the lower side).

\section{Initial findings and the questions emerged}

The simulations are run until a non-dimensional time of $t a_{\infty} / L_{c}=20$, which is sufficient to capture both the fluctuating wall pressure signals and the far field sound. For the subsequent sections the following quantities are used repeatedly. Fluctuating acoustic and wall pressures are denoted by $p_{a}^{\prime}$ and $\Delta p_{w}$, respectively, defined here as

$$
p_{a}^{\prime}(\boldsymbol{x}, t)=p_{a}(\boldsymbol{x}, t)-p_{\infty}, \quad \Delta p_{w}(\boldsymbol{x}, t)=p_{w}\left(x, y=0^{+}, z, t\right)-p_{w}\left(x, y=0^{-}, z, t\right),
$$

where the subscripts $y=0^{+}$and $0^{-}$indicate the upper and lower surfaces of the flat plate aerofoil, respectively. The wall pressure fluctuation $\left(\Delta p_{w}\right)$ defined in this paper is therefore representing the aeroacoustic source strength. The power spectral density (PSD) of the wall pressure fluctuation can then be determined as

$$
S_{p p w}(\boldsymbol{x}, f)=2 \widehat{\Delta p_{w}}(\boldsymbol{x}, f){\widehat{\Delta p_{w}}}^{*}(\boldsymbol{x}, f),
$$

where " $\wedge$ " represents a Fourier transformed variable and " $*$ " denotes a complex conjugate. For the subsequent analysis surface quantities are extracted one grid point aft of the leading edge, where the largest pressure fluctuation is obtained in the current simulations. Similarly the PSD of the acoustic pressure may be determined as

$$
S_{p p a}(\boldsymbol{x}, f)=2 \widehat{p_{a}^{\prime}}(\boldsymbol{x}, f){\widehat{p_{a}^{\prime}}}^{*}(\boldsymbol{x}, f)
$$

which is calculated at an observer location $\boldsymbol{x}_{o}=\left(0,5 L_{c}, 0\right)$ in the present study. The sound attenuation due to a WLE relative to the SLE case is then quantified by

$$
\Delta S P L(\boldsymbol{x}, f)=10 \log _{10}\left[\frac{\left.S_{p p a}(\boldsymbol{x}, f)\right|_{\mathrm{SLE}}}{\left.S_{p p a}(\boldsymbol{x}, f)\right|_{\mathrm{WLE}}}\right] .
$$

\subsection{Wall and acoustic pressure fluctuations}

As a result of the current simulations, figure 4 shows the time signals and corresponding PSD (power spectral density) functions of the wall pressure fluctuation $\left(\Delta p_{w}\right)$ on the leading edge points for three different WLE geometries $\left(h_{L E} / L_{c}=1 / 30,1 / 15\right.$ and $\left.1 / 10\right)$ compared with the SLE baseline case. First, it is apparent in the figure that the level of wall pressure fluctuation (aeroacoustic source strength) at the hill location constantly decreases with increasing $h_{L E}$ (WLE amplitude) across the entire frequency range. This is directly related to the "source cutoff" effect due to the geometric obliqueness as suggested by Kim et al. (2016), i.e. $\Delta p_{w} \propto \cos \theta$ where $\theta$ is the local sweep angle of the leading edge. Figure 4 also shows that the source strength at the root does not seem to change much with $h_{L E}$ (maintained at the level of the SLE case), which might be rather simply anticipated because the sweep angle is locally zero at the root. However, at the peak where the sweep angle is also zero, the source strength significantly changes with $h_{L E}$ although it does not drop as consistently as the hill case and seems to converge towards a constant level. The distinction in source strength between the peak and root was also reported by Kim et al. (2016) with little understood about its cause. Therefore, the main focus of the paper is on understanding and explaining the disparity in source behaviours between the peak and root.

Figure 5 shows the far-field radiated acoustic pressure signals, their PSDs and the relative noise reduction spectra for the three different WLE amplitudes as tested in figure 4. The reduction of the sound pressure level in the WLE cases is clear in this 

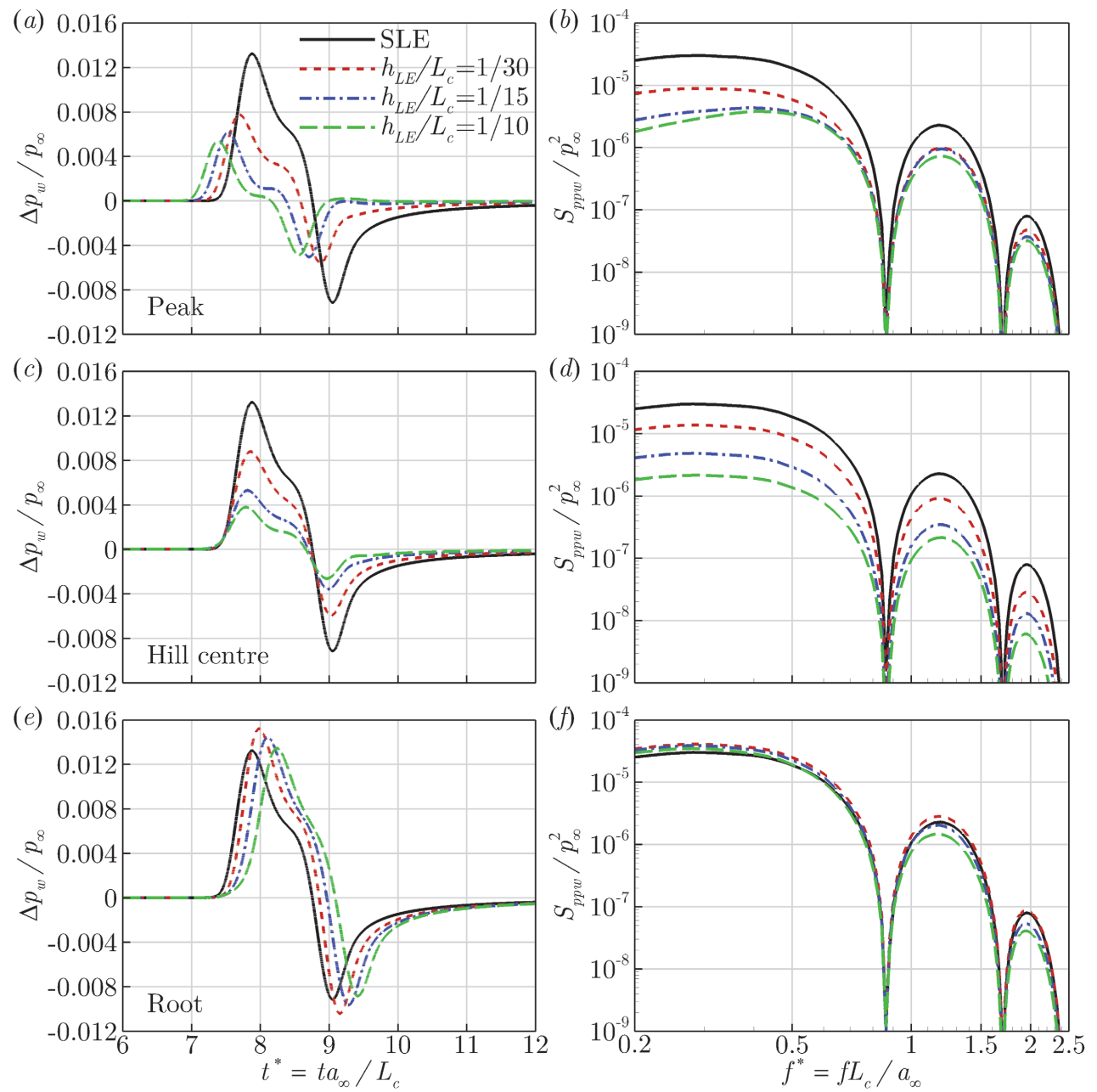

FiguRE 4. Fluctuating wall pressure time signals, $\Delta p_{w}\left(t^{*}\right) / p_{\infty}$, and the corresponding power spectral density (PSD), $S_{p p w}\left(f^{*}\right) / p_{\infty}^{2}$, obtained at three different locations on the WLE (peak, hill centre and the root) for three different values of the WLE amplitude $\left(h_{L E} / L_{c}=1 / 30,1 / 15\right.$ and $1 / 10)$ with its wavelength fixed $\left(\lambda_{L E} / L_{c}=2 / 15\right)$, compared with the SLE baseline case.

figure. It is also apparent that the noise reduction increases with the WLE amplitude $\left(h_{L E}\right)$ and with the frequency as well. The same observations have been made in the previous studies with various upstream flow conditions as mentioned in $\S 1$ (Lau et al. 2013; Clair et al. 2013; Narayanan et al. 2015; Chaitanya et al. 2015; Kim et al. 2016). The current vortex flow condition adds another example in which the WLE performs successfully for noise reduction. However, it is still unknown as to how the radiated sound field achieves such a large reduction in the high frequency range (figure $5 b$ ) when the source strength at the peak and root does not drop as much in the same frequency range (figure $4 b$ and $4 f$ ). On the contrary, the substantially weakened source strength in the low frequency range at the peak and hill (figure $4 b$ and $4 d$ ) does not seem to project on the radiated sound field at all (figure $5 b$ ). It is speculated that there exist some crucial mechanisms particularly in the propagation process leading to these controversial results. The authors stress that substantial work is required in order to understand the hidden 


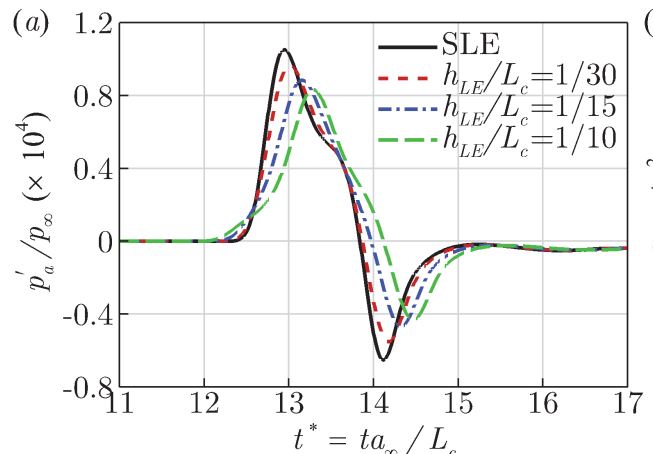

$(c)$

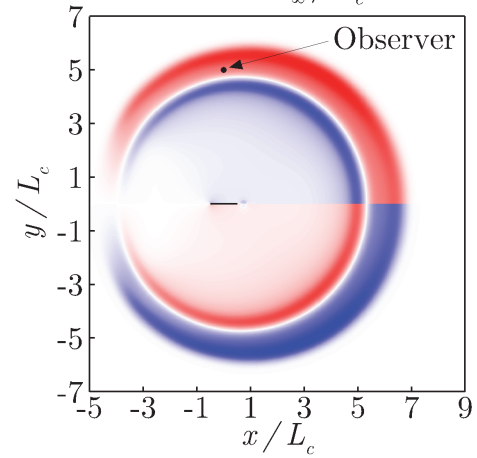

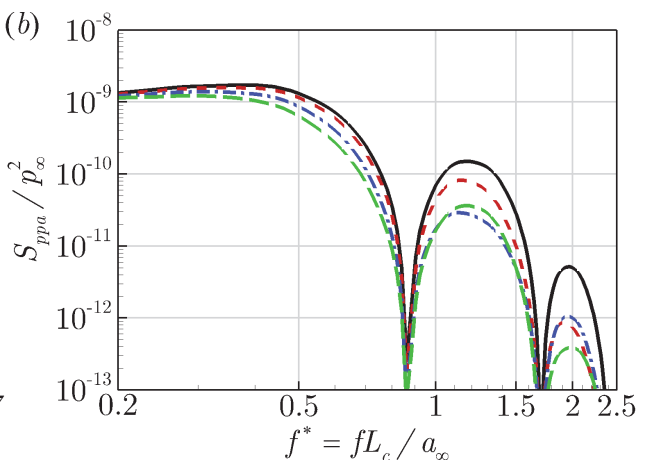

$(d)$

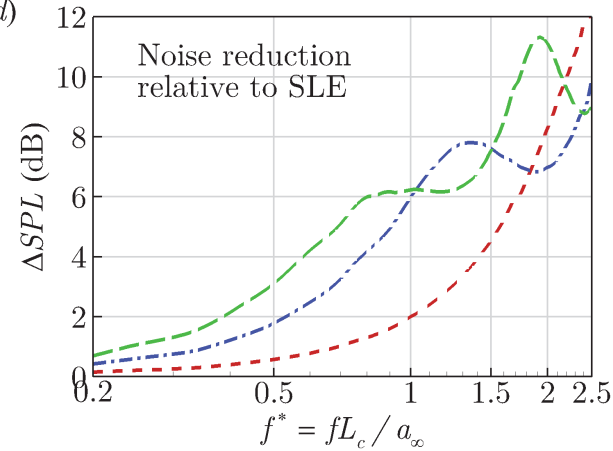

FiguRE 5. Far-field acoustic pressure time signals, $p_{a}^{\prime}\left(t^{*}\right) / p_{\infty}$, the corresponding PSD, $S_{p p a}\left(f^{*}\right) / p_{\infty}^{2}$, and the relative noise reduction spectra, $\triangle S P L\left(f^{*}\right)$, obtained at an observer location, $\boldsymbol{x}_{o}=\left(0,5 L_{c}, 0\right)$, from three different WLE geometries $\left(h_{L E} / L_{c}=1 / 30,1 / 15\right.$ and $1 / 10$ with $\left.\lambda_{L E} / L_{c}=2 / 15\right)$, compared with the SLE baseline case.

propagation mechanisms. This paper is yet focused on the source mechanisms which need to be established beforehand.

\subsection{Horseshoe vortex systems induced by $W L E$}

As mentioned above, the distinction between the peak and root source behaviours seems contradictory to some extent, as one would assume that both interact with the impinging vortex in a parallel fashion (zero geometric obliqueness). The authors find that the distinction is mainly attributed to the dynamics of secondary vortices created along the WLE. The impinging vortex (rotating clockwise viewed from the $x y$-plane) induces a downwash downstream of its core and consequently an upwash upstream of it as indicated in figure 3. As the vortex moves close to the aerofoil with a SLE the preceding downwash $(v<0)$ is suppressed very rapidly to zero $(v=0)$ at the leading edge due to the no-penetration (wall) condition. The upshot of this is that a high velocity gradient $(\partial v / \partial x \gg 0$ while $\partial u / \partial y \approx 0)$ is created at the leading edge, which results in a counter-clockwise spanwise vorticity there $\left(\omega_{z}=\partial v / \partial x-\partial u / \partial y \gg 0\right)$. This is a typical example of vortex-body interaction (Rockwell 1998). In the SLE case the induced spanwise vorticity $\left(\omega_{z}\right)$ is uniform along the span. However, in a WLE case, $\omega_{z}$ varies along the span and also a streamwise vorticity $\left(\omega_{x}\right)$ is produced due to a significant $\partial v / \partial z$ created at the hill side via the same mechanism described above.

Figure 6 shows snapshots of the WLE-vortex interaction taken as time elapses from the first contact until the vortex completely detaches from the WLE. The vortex travelling through the aerofoil is shown by the spanwise vorticity contour surfaces $\left(\omega_{z} L_{c} / a_{\infty}\right)$ and 


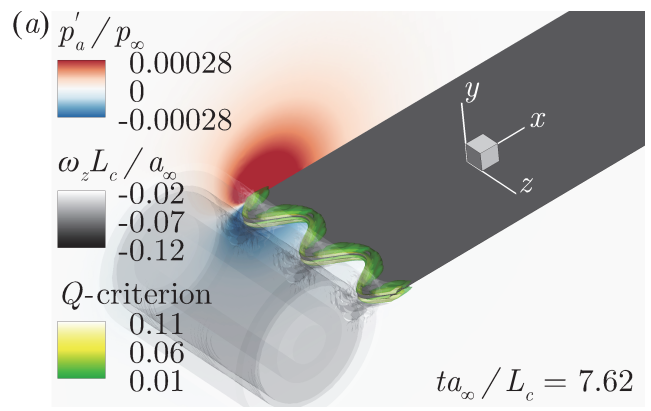

(b)

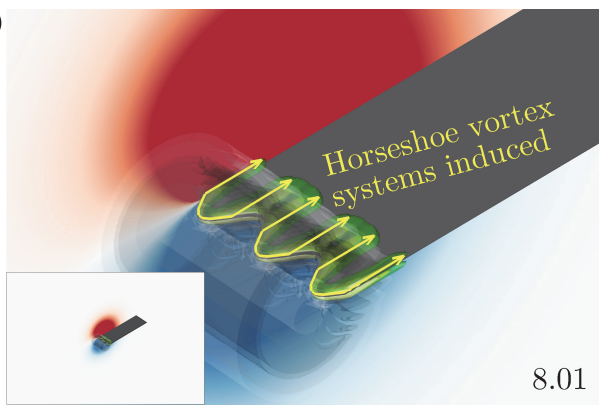

(c)
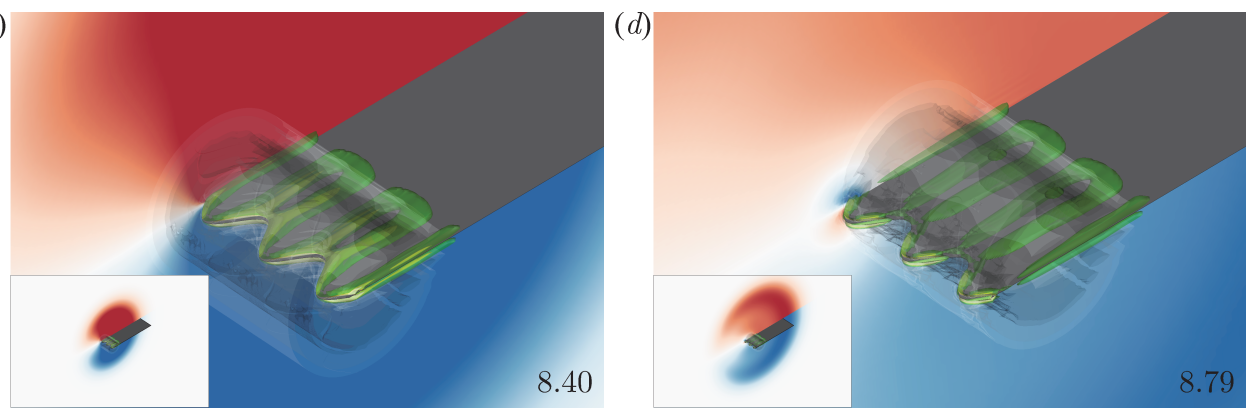

(e)

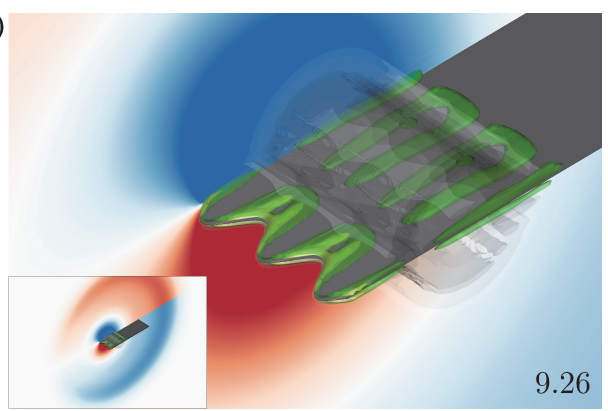

$(f)$



FiguRE 6. Snapshots of WLE-vortex interaction in temporal order: contour plots for perturbed acoustic pressure $\left(p_{a}^{\prime} / p_{\infty}\right)$, spanwise vorticity $\left(\omega_{z} L_{c} / a_{\infty}\right)$ and the $Q$-criterion $\left(\left\|\Omega_{i j} L_{c} / a_{\infty}\right\|^{2}-\left\|S_{i j} L_{c} / a_{\infty}\right\|^{2}\right.$ where $\Omega_{i j}$ and $S_{i j}$ are the vorticity and rate-of-strain tensors, respectively). The figures show secondary vortices ( $Q$-criterion) and sound waves $\left(p_{a}^{\prime} / p_{\infty}\right)$ generated at the leading edge during the interaction. The bottom left corner of images $(b)-(f)$ provides a zoomed out perspective visualising the radiating sound waves. $h_{L E} / L_{c}=1 / 30$.

the induced secondary vortices on the wall are visualised by using the $Q$-criterion contour surfaces. In addition, the plots of $p_{a}^{\prime} / p_{\infty}$ in the $x y$-plane show the dipole sound pulses with an alternating sign being generated when the downwash and upwash strokes of the travelling vortex impact the leading edge.

One of the most critical features shown in figure 6 is the creation of horseshoelike vortex systems emanating from the WLE as shown from the $Q$-criterion plots. The horseshoe vortex system consists of a bound vortex sitting at the WLE around the peak and two counter-rotating streamwise vortices trailing from the bound vortex as depicted in figure $6 b$. The horseshoe vortex systems are created twice during the downwash and upwash strokes, and the direction of the rotation reverses between them. The gap between the pair of streamwise vortices becomes larger as the initial vortex moves further downstream. However, this results in a decreasing gap between two neighbouring 
(a) $t a_{\infty} / L_{c}=8.01$

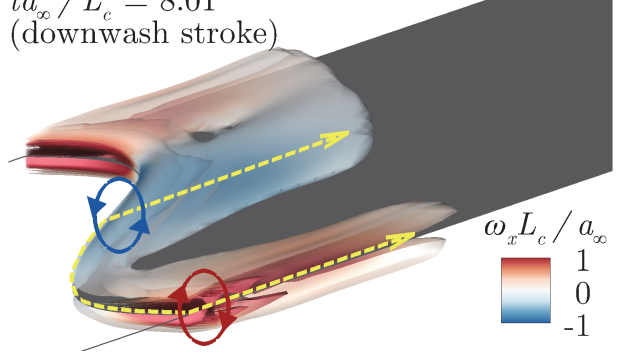

(b) $t a_{\infty} / L_{c}=9.26$

(upwash stroke)

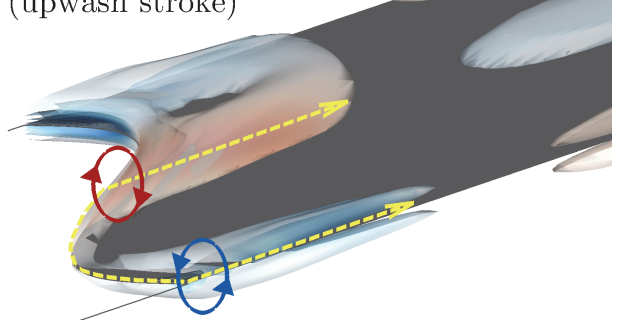

FIgURE 7. $Q$-criterion iso-surfaces coloured by streamwise vorticity $\left(\omega_{x} L_{c} / a_{\infty}\right)$ taken at two different points in time during $(a)$ the downwash and $(b)$ upwash strokes of the impinging vortex. The red and blue surfaces indicate positive (clockwise) and negative (anti-clockwise) rotations around the $x$-axis, respectively. The dashed yellow curves indicate the WLE-induced horseshoe vortex systems.

(a)

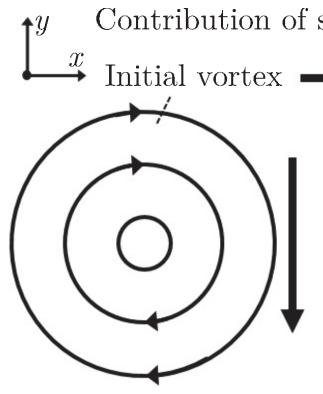

spanwise vorticity $\left(\omega_{z}\right)$

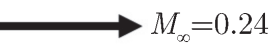

WLE induced vortex

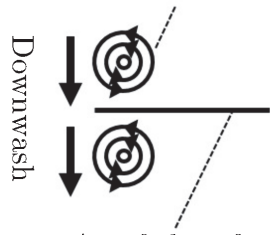

Aerofoil surface (b)

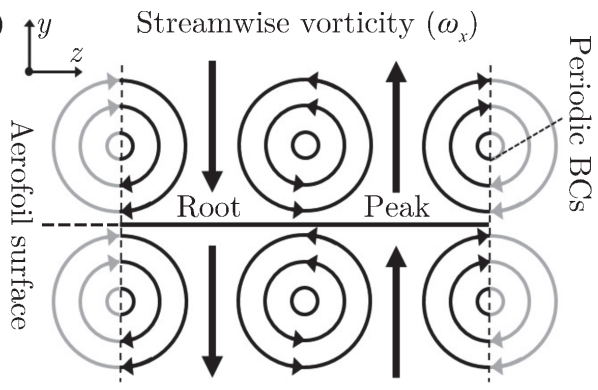

FiguRE 8. Simplified illustration of the WLE-induced vorticity distribution exhibited in Figures 6 and 7: $(a)$ the sideview on the WLE showing the contribution of spanwise vorticity components induced during the downwash stroke of the impinging vortex; and, $(b)$ the frontview on the WLE describing the streamwise vorticity contribution creating an upwash at the peak and a downwash at the root as a result.

streamwise vortices from two adjacent horseshoe vortex systems. These are significantly different vortex dynamics, which do not appear in the SLE case.

In figure 7 the $Q$-criterion contour surfaces shown in figures $6 b$ and $6 e$ are recoloured by the streamwise vorticity $\left(\omega_{x} L_{c} / a_{\infty}\right)$ indicating the direction of rotation about the $x$-axis. A brief sketch of this event is provided in figure 8 depicting the horseshoevortex-induced vorticity distributions at a single instant of time during the downwash stroke of the impinging vortex. It is illustrated in the figure that, during the downwash stroke, the horseshoe-vortex-induced streamwise vorticity components create an additional downwash at the root but a counteracting upwash at the peak (figure $8 b$ ). For the upwash stroke the opposite trend takes place. On a minor note, the streamwise vorticity distribution observed from the simulation data is almost symmetrical across both sides of the aerofoil with a minor difference in magnitudes.

It is reasonable to assume that the aeroacoustic source strength is almost purely governed by the vertical velocity component (downwash and upwash) impinging on the leading edge since the current aerofoil geometry is completely horizontal with zero thickness and viscosity. Based on this assumption, the authors suggest that the uneven spanwise distribution of the induced vertical velocity illustrated in figure $8 b$ is mainly responsible for the disparity in source behaviours between the peak and root observed in $§ 3.1$. It is apparent in the figure $8 b$ that the peak is experiencing a reduced level of 


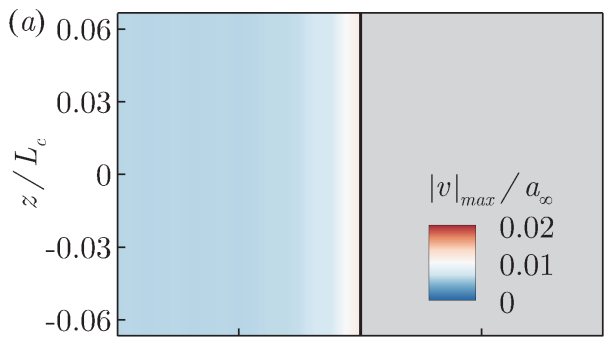

(b)
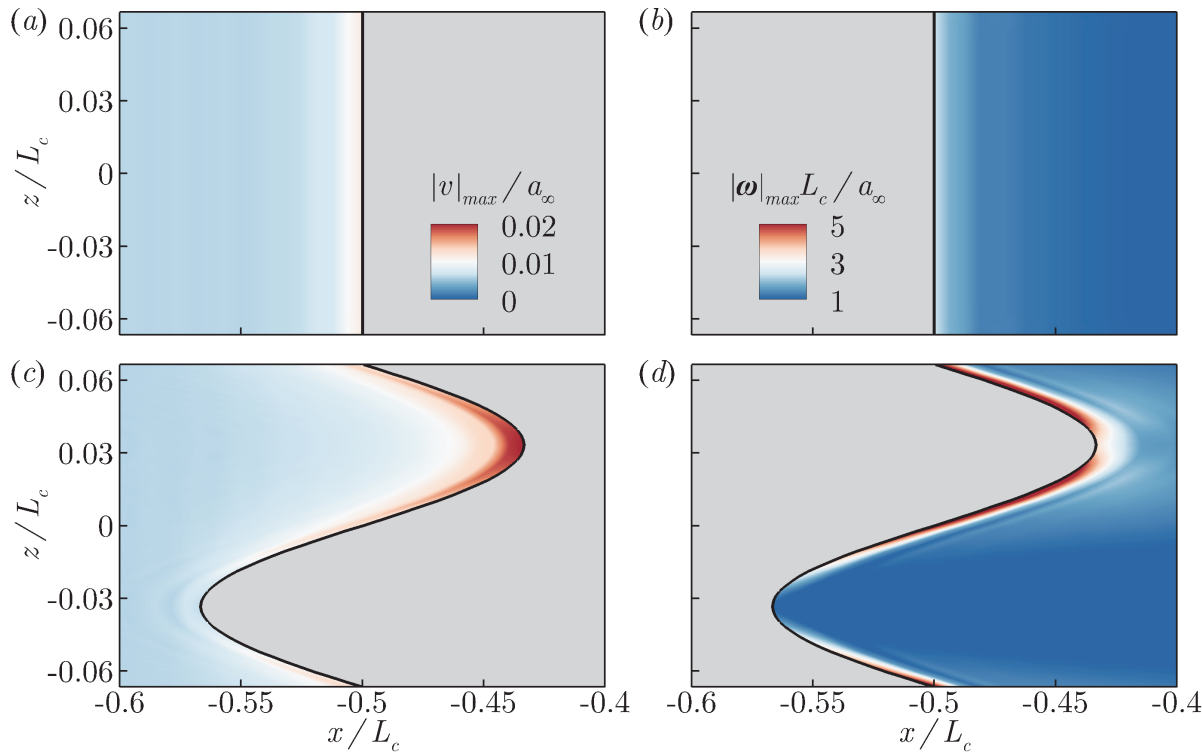

FiguRE 9. The distribution of maximum values (taken from time signals) of the perturbed vertical velocity $\left(|v|_{\max } / a_{\infty}\right)$ and the vorticity magnitude $\left(\left.|\boldsymbol{\omega}|\right|_{\max } L_{c} / a_{\infty}\right)$ on the horizontal plane $(y=0):(a / b)$ for a SLE baseline case and $(c / d)$ for a WLE case with $h_{L E} / L_{c}=1 / 15$. The vertical velocity plots are shown in the flow field only, and the vorticity magnitude plots on the aerofoil's upper surface only.

vertical velocity perturbation due to the counteracting effect of the streamwise vortices, hence a weaker source strength at the peak as seen in figures $4 a$ and $4 b$. On the other hand, the root experiences an amplified level of the vertical velocity perturbation and therefore a reinforced source strength at the root, which is also manifested to a certain extent in figures $4 e$ and $4 f$ (at low frequencies). However, the relative difference to the baseline SLE case is much smaller at the root compared to that taking place at the peak. This requires an explanation. Additional quantitative investigations provided in the following sections reveal further details of the source behaviours.

\section{Consistent source strength at the root}

Figure 9 shows the distribution of the maximum values of the net induced vertical velocity $\left(|v|_{\max } / a_{\infty}\right)$ and vorticity magnitude $\left(|\boldsymbol{\omega}|_{\max } L_{c} / a_{\infty}\right)$ on the horizontal plane $(y=0)$ where the aerofoil is located, obtained from each time signal at every point in space:

$$
|v|_{\max }(\boldsymbol{x})=\max _{t=0}^{\infty}|v(\boldsymbol{x}, t)|, \quad|\boldsymbol{\omega}|_{\max }(\boldsymbol{x})=\max _{t=0}^{\infty}|\boldsymbol{\omega}(\boldsymbol{x}, t)| .
$$

It can be seen in figure $9 c$ that the amplitude of the induced vertical velocity is highest around the WLE root area as expected. It is also indicated in figure $9 d$ that the streamwise vortices once created at the peak become amplified as they are moving downstream along the hill towards the root, which results in the highly concentrated vertical velocity spot around the root as anticipated from figure $8 b$. These are, surprisingly, much higher (by a factor of two) than those of the SLE baseline cases, which leads to a question how such a highly amplified level of velocity perturbation at the root settles down with a minor increase in the aeroacoustic source strength (wall pressure fluctuation) as seen in figures $4 e$ and $4 f$. A discussion on this follows below. 

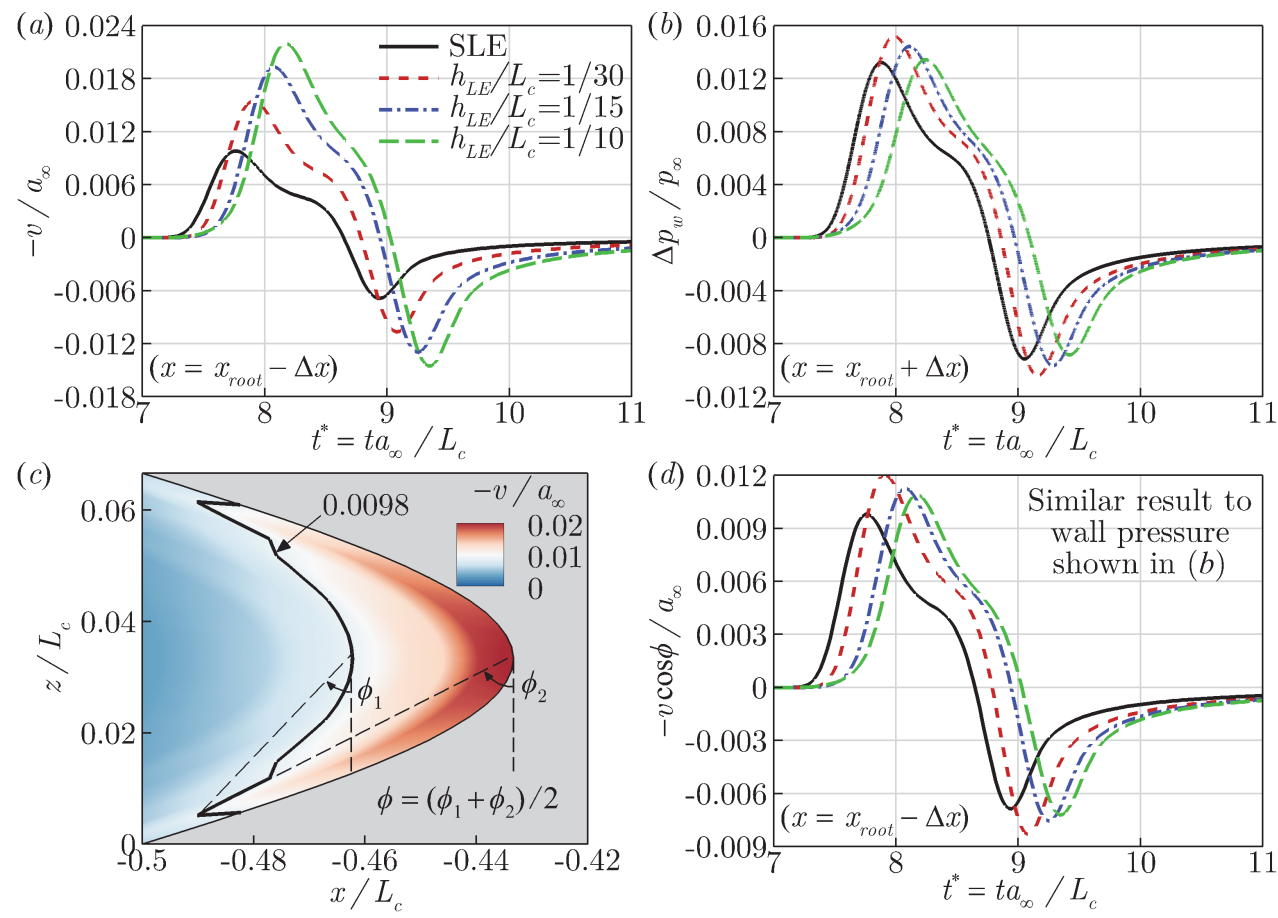

FiguRE 10. A comparison between the induced vertical velocity and wall pressure fluctuation $\left(v / a_{\infty}\right.$ and $\left.\Delta p_{w} / p_{\infty}\right)$ at the WLE root for various WLE amplitudes $\left(h_{L E} / L_{c}\right):(a)$ time signals of $v / a_{\infty}$ obtained one grid point upstream of the root; $(b)$ time signals of $\Delta p_{w} / p_{\infty}$ obtained one grid point downstream of the root; $(c)$ an instantaneous contour plot of $v / a_{\infty}$ taken at $t^{*}=8.08$ (when the induced vertical velocity reaches its maximum, for $h_{L E} / L_{c}=1 / 15$ ) indicating an oblique interaction with the root, where the values greater than 0.0098 indicate stronger perturbation than the SLE case; and, $(d)$ time signals of $v \cos \left[\left(\phi_{1}+\phi_{2}\right) / 2\right] / a_{\infty}$ which includes the obliqueness effect. The values of $\phi_{1}$ and $\phi_{2}$ are listed in table 1 .
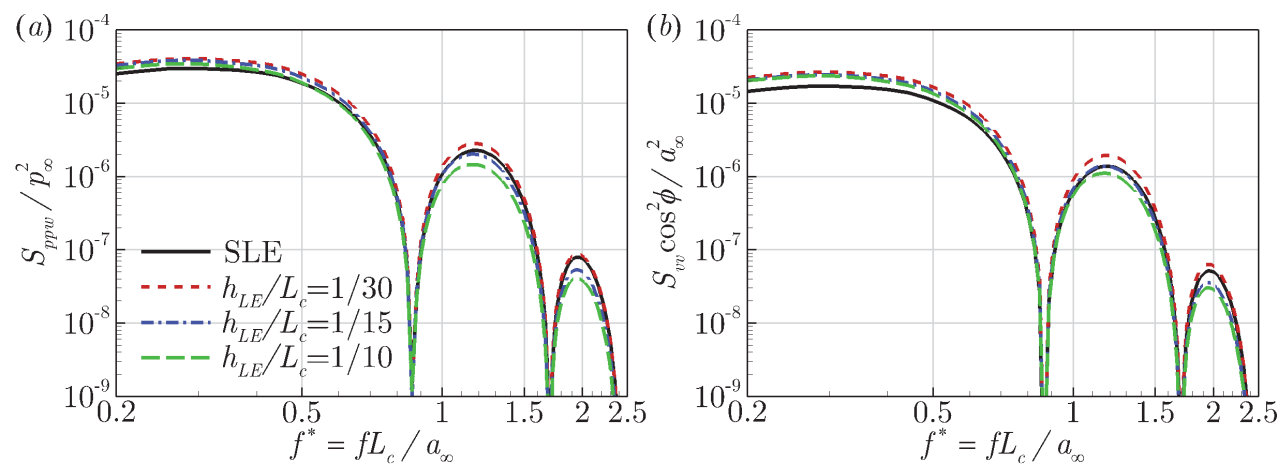

FIGURE 11. Spectral similarity at the root between the wall pressure fluctuation and the induced vertical velocity including the obliqueness effect: ( $a$ ) PSD of $\Delta p_{w} / p_{\infty}$ and (b) PSD of $v \cos \phi / a_{\infty}$ from figure $10 b$ and $10 d$, respectively.

The time signals of the induced vertical velocity impinging at the root are provided in figure $10 a$ for various values of $h_{L E}$, where it is clear that the velocity perturbation increases significantly and steadily with $h_{L E}$ (linked with the streamwise vorticity intensifying as the WLE becomes slender). On the contrary, the wall pressure fluctuation at 


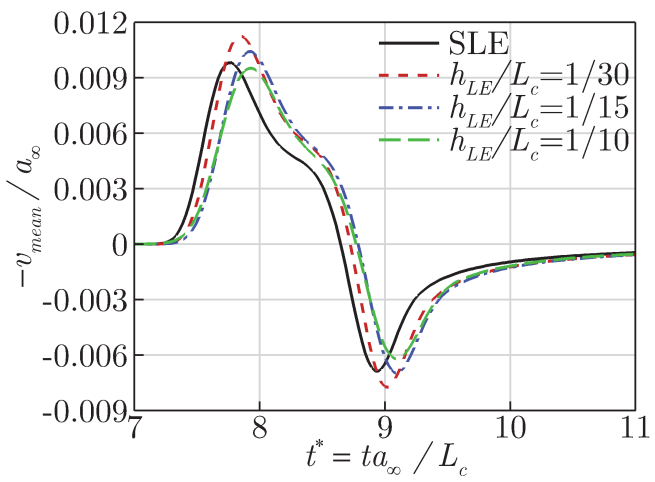

FiguRE 12. Mean vertical velocity signals calculated from (4.2) around the root area for various values of $h_{L E} / L_{c}$ (WLE amplitude), in relation to the consistent source strength at the root irrespective of $h_{L E}$.

the root shown in figure $10 \mathrm{~b}$ does not vary as much, and even slightly decreases with increasing $h_{L E}$. One of the possible answers to this paradoxical event might be related to the fact that the vertical velocity perturbation approaches the root area in an oblique fashion as can be seen from figure $10 c$. The vertical velocity iso-contours that are skewed and bent around the root area exhibit the oblique interaction taking place. In order to quantify the obliqueness of the interaction, as shown in figure $10 c$, two cosine angles $\left(\phi_{1}\right.$ and $\left.\phi_{2}\right)$ are measured from the slender crescent-shaped high-intensity area in which the vertical velocity perturbation is stronger than the maximum value of the SLE case $\left(-v / a_{\infty}=0.0098\right)$. The angles $\phi_{1}$ and $\phi_{2}$ are defined between the tip and the two inflection points of the crescent contour line. The authors then take the average of the two measures to represent the overall obliqueness, i.e. $\phi=\left(\phi_{1}+\phi_{2}\right) / 2$. The values of these angles estimated for each of the cases $\left(h_{L E} / L_{c}=1 / 30,1 / 15\right.$ and $\left.1 / 10\right)$ are listed in table 1 . The authors have examined the sensitivity of $\phi$ for various values of the threshold (up to $\pm 20 \%$ from 0.0098) and found no significant changes as shown in Appendix B. Figure $10 d$ reveals that the vertical velocity signals multiplied by the obliqueness factor $(\cos \phi)$ are remarkably similar to the wall pressure fluctuation signals $\left(\Delta p_{w} / p_{\infty}\right)$ given in figure $10 \mathrm{~b}$. They even show the same trend of changes with respect to increasing $h_{L E}$. The remarkable similarity is also demonstrated in the spectral domain in figure 11 where the agreement is consistent throughout the entire frequency range. The result here might strongly suggest that the oblique interaction is one of the possible explanations of the consistent source strength at the root.

\begin{tabular}{cccc}
\hline$h_{L E} / L_{c}$ & $\phi_{1}$ & $\phi_{2}$ & $\phi=\frac{\phi_{1}+\phi_{2}}{2}$ \\
\hline $1 / 30$ & $30.13^{\circ}$ & $47.72^{\circ}$ & $38.93^{\circ}$ \\
$1 / 15$ & $45.37^{\circ}$ & $63.33^{\circ}$ & $54.35^{\circ}$ \\
$1 / 10$ & $50.76^{\circ}$ & $69.76^{\circ}$ & $60.26^{\circ}$ \\
\hline
\end{tabular}

TABLE 1. The cosine angles representing the oblique interaction between the induced vertical velocity and the WLE shown in figure $10 c$.

An additional investigation is performed in this section to find out that there is a certain normalisation applicable to the vertical velocity perturbation, which leads to a 
meaningful correlation with the wall pressure fluctuation at the root. In this regard, an area-averaged norm of the vertical velocity perturbations is defined around the root as a function of time as follows:

$$
\left.\begin{array}{rl}
v_{\text {mean }}(t) & =\frac{1}{\mathcal{A}} \int_{0}^{\frac{1}{2} \lambda_{L E}} \int_{-\frac{1}{2} L_{c}}^{x_{L E}} v(x, y=0, z, t) \mathrm{d} x \mathrm{~d} z, \\
\mathcal{A} & =\int_{0}^{\frac{1}{2} \lambda_{L E}} \int_{-\frac{1}{2} L_{c}}^{x_{L E}} \mathrm{~d} x \mathrm{~d} z=\frac{\lambda_{L E} h_{L E}}{\pi},
\end{array}\right\}
$$

where $\mathcal{A}$ is the area of integration enclosed by the concave part of the WLE sinusoid towards the root and a straight line where the SLE was positioned (e.g. the concave area shown in figure 10c). The expression for $x_{L E}$ as a function of $z$ is given in (2.2). The calculated mean vertical velocity signals are presented in figure 12 . It is shown that the mean vertical velocity perturbation remains relatively unchanged despite the substantial variation in $h_{L E}$. This might indicate that the overall vertical momentum (created by the initial vortex) is conserved although the vertical velocity tends to surge locally around the root area. The $v_{\text {mean }} / a_{\infty}$ signals also exhibit a good similarity with the $\Delta p_{w} / p_{\infty}$ (wall pressure fluctuation) signals at the root shown in figure $10 b$. Therefore, it may be concluded here that the source strength at the root is better correlated with the mean vertical velocity signal than with the local one nearest to it. This outcome is analogous to the theoretical consideration based on a Poisson equation, i.e. $\nabla^{2} p=$ $-\rho\left(\partial u_{i} / \partial x_{j}\right)\left(\partial u_{j} / \partial x_{i}\right)$ - see Tsuji et al. (2007) - indicating that pressure at a certain location is determined by integrating velocity properties surrounding it.

\section{Variations in source strength at the peak}

The focus is now moved on to the WLE peak in this section. It has been observed earlier in $\S 3$ that the aeroacoustic source characteristics at the peak are considerably different to those at the root. The source strength at the peak decreases significantly with increasing WLE amplitude $\left(h_{L E}\right)$ as shown in figure $4 a$ and $4 b$. It seemed rather straightforward from figure $8 b$ that this was due to the attenuating vertical velocity contribution created at the peak by the streamwise vorticity components that are growing with $h_{L E}$ as shown in figures 13 and 14. The significant growth of the streamwise vorticity appears counterbalanced by a decay in the spanwise vorticity to a certain extent, which corresponds to Helmholtz's theorems. The time signals of the induced vertical velocity and the corresponding wall pressure fluctuation at the peak varying with $h_{L E}$ are shown in figure 15. It is apparent in the figure that the vertical velocity perturbation decreases with increasing $h_{L E}$ and it results in the reduction of the source strength at the peak. A more interesting observation, however, is that both $v$ and $\Delta p_{w}$ become significantly less sensitive to increasing $h_{L E}$ after a certain point. In particular the $v$ signal seems to converge towards that of the free-field solution generated without the aerofoil in place. This section is focused on explaining the mechanism of the inconsistent source behaviours taking place at the peak.

Considering the horseshoe vortex (HV) system identified in $\S 3$, a semi-analytic approach based on Biot-Savart's law is proposed in order to obtain more information on the induced velocity components from the HV system. The general form of Biot-Savart's law is expressed by

$$
\mathrm{d} \boldsymbol{v}\left(\boldsymbol{x}_{0}, \boldsymbol{l}\right)=\frac{\Gamma}{4 \pi} \frac{\boldsymbol{r} \times \mathrm{d} \boldsymbol{l}}{|\boldsymbol{r}|^{3}}, \quad \boldsymbol{r}=\boldsymbol{l}-\boldsymbol{x}_{0},
$$

where $\boldsymbol{x}_{0}$ and $\boldsymbol{l}$ are position vectors of the observer and a certain point on the vortex 


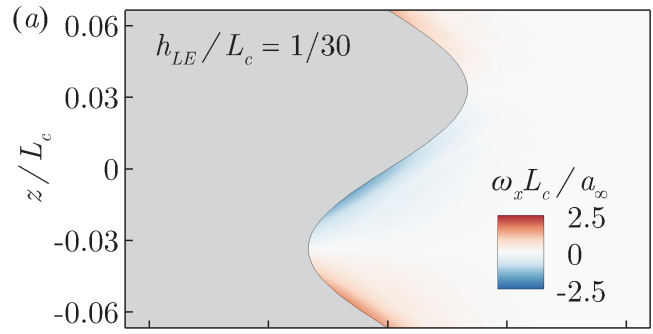

(b)


(d)
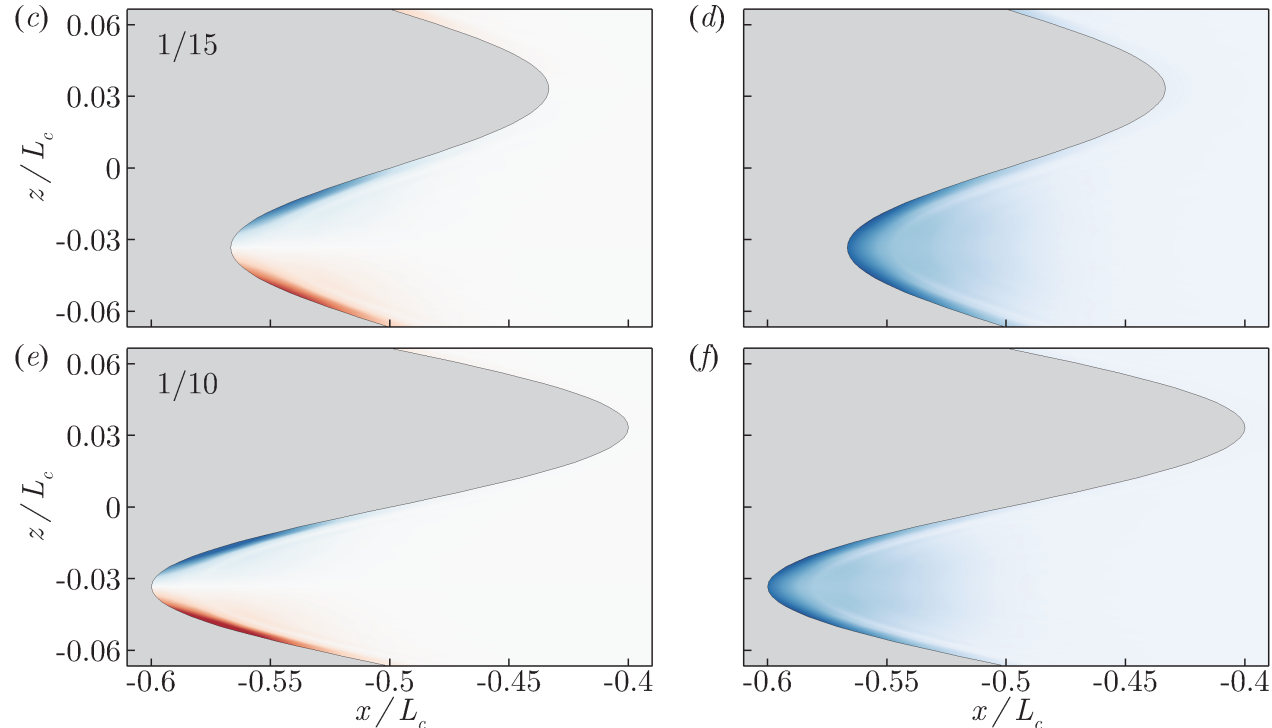

$(f)$

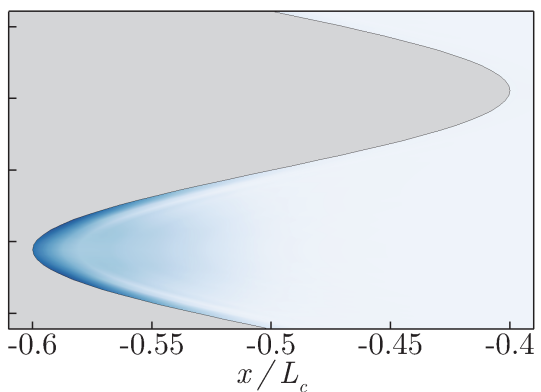

FiguRE 13. Instantaneous contour plots of streamwise vorticity $\left(\omega_{x} L_{c} / a_{\infty}\right.$ : left $)$ and spanwise vorticity $\left(\omega_{z} L_{c} / a_{\infty}\right.$ : right) on the upper surface of the aerofoil for three different WLE amplitudes, obtained when the induced vertical velocity in front of the peak reaches its maximum amplitude: $(a / b) t a_{\infty} / L_{c}=7.540,(c / d) 7.461$ and $(e / f) 7.305-$ see figure $15 a$.
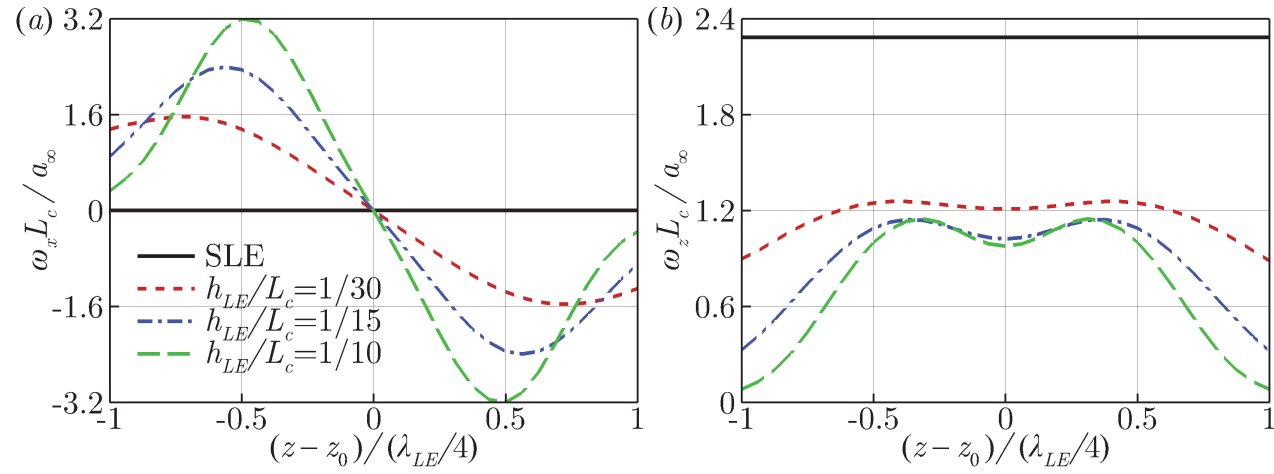

FigURE 14. Vorticity distributions in span along the leading edge for various values of $h_{L E} / L_{c}$ obtained when the induced vertical velocity in front ot the peak reaches its maximum amplitude: (a) streamwise vorticity $\left(\omega_{x} L_{c} / a_{\infty}\right)$ and $(b)$ spanwise vorticity $\left(\omega_{z} L_{c} / a_{\infty}\right)$ on the upper surface of the aerofoil - see figure 13 . The spanwise coordinate of the peak is denoted by $z_{0}$ in the $x$-axis labels. 

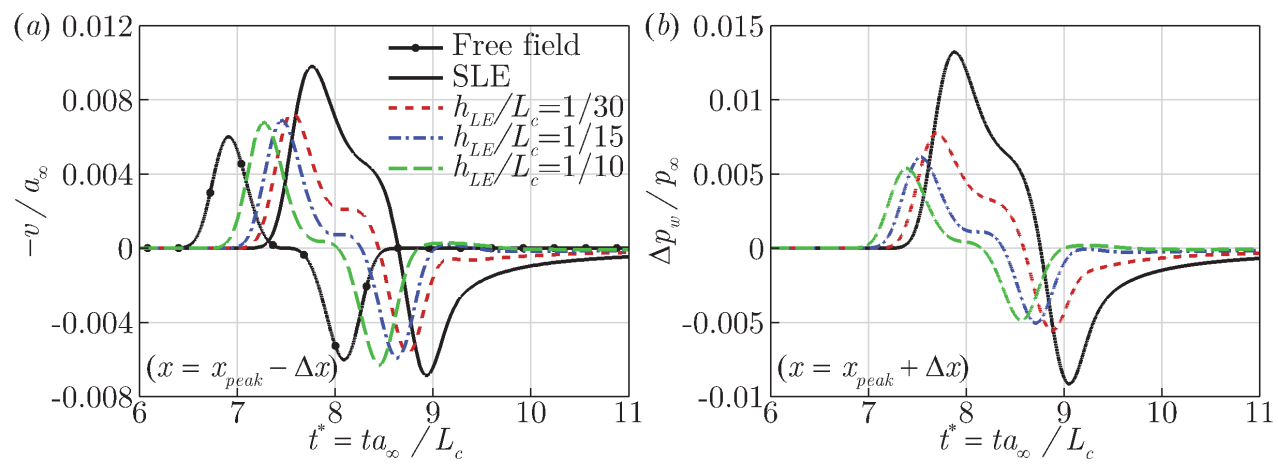

Figure 15. A comparison between the induced vertical velocity and wall pressure fluctuation $\left(v / a_{\infty}\right.$ and $\left.\Delta p_{w} / p_{\infty}\right)$ at the WLE peak for various WLE amplitudes $\left(h_{L E} / L_{c}\right):(a)$ time signals of $v / a_{\infty}$ obtained one grid point upstream of the peak; and, (b) time signals of $\Delta p_{w} / p_{\infty}$ obtained one grid point downstream of the peak. The case with "Free field" indicates a free-field solution (without the aerofoil) recorded at $(x, y)=\left(-0.7 L_{c}, 0\right)$.

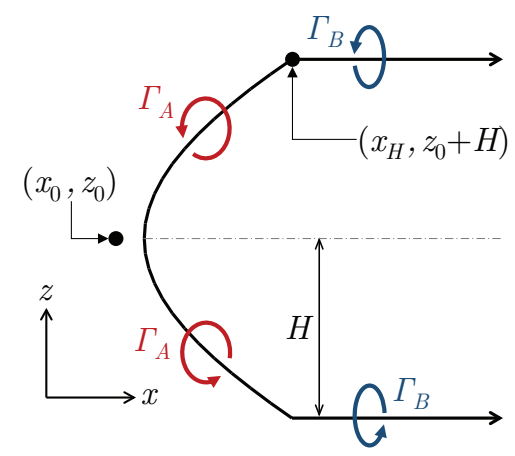

FiguRE 16. Schematic of the horseshoe vortex (HV) system used to estimate the WLE-induced vertical velocity in front of the peak, in relation to (5.3).

filament, respectively; and, $\Gamma$ is the cross-sectional circulation induced by the vortex. Since the current HV (horseshoe vortex) system is on a horizontal plane, the above equation with the observer position fixed at the peak can be simplified as

$$
\mathrm{d} v_{\text {peak }}^{H V}=\frac{\Gamma}{4 \pi} \frac{\left(r_{3} \mathrm{~d} x-r_{1} \mathrm{~d} z\right)}{\left(r_{1}^{2}+r_{3}^{2}\right)^{\frac{3}{2}}},
$$

where the subscripts " 1 " and " 3 " denote the streamwise and spanwise coordinates, respectively. Integrating all contributions from the bound vortex and the two trailing vortices lead to the following formula (see figure 16):

$$
\left.\begin{array}{c}
v_{\text {peak }}^{H V}=v_{A}+v_{B}, \\
v_{A}=\frac{1}{4 \pi} \int_{z_{0}-H}^{z_{0}+H} \frac{\Gamma_{A}}{\left[\left(x_{L E}-x_{0}\right)^{2}+\left(z-z_{0}\right)^{2}\right]^{\frac{3}{2}}}\left[\left(z-z_{0}\right) \frac{\mathrm{d} x_{L E}}{\mathrm{~d} z}-\left(x_{L E}-x_{0}\right)\right] \mathrm{d} z, \\
v_{B}=\frac{H}{2 \pi} \int_{x_{H}}^{\infty} \frac{\Gamma_{B} \mathrm{~d} x}{\left[\left(x-x_{0}\right)^{2}+H^{2}\right]^{\frac{3}{2}}},
\end{array}\right\}
$$

where the subscripts " $A$ " and " $B$ " represent contributions from the bound and trailing vortices, respectively; $\left(x_{0}, z_{0}\right)=\left(-L_{c} / 2-h_{L E}-\Delta x,-\lambda_{L E} / 4\right)$ is the observer position 

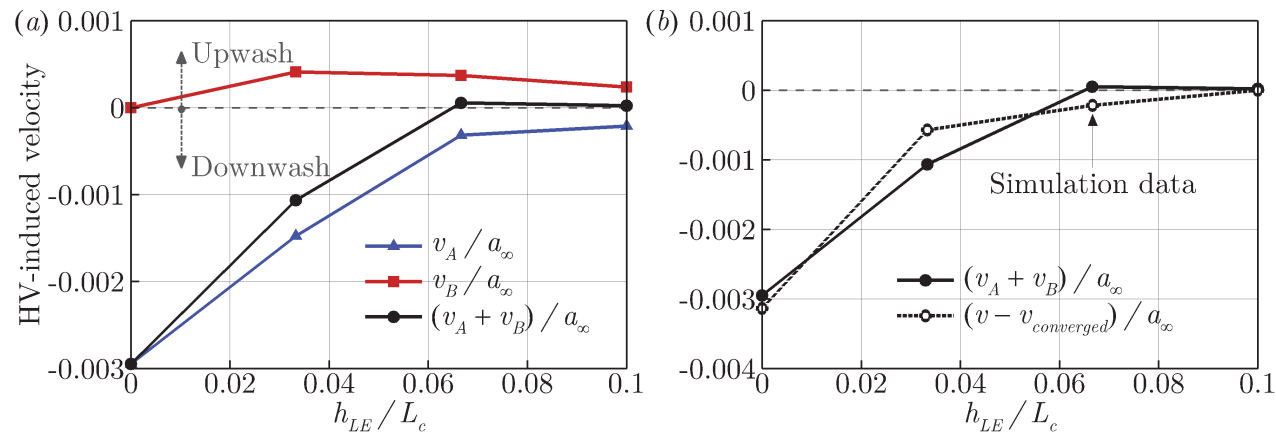

Figure 17. Semi-analytic predictions of the HV-induced vertical velocity components in front of the peak, obtained by using (5.3) for various values of $h_{L E} / L_{c}:(a)$ contributions from the bound vortex $\left(v_{A}\right)$ and the trailing vortices $\left(v_{B}\right)$, and the total $\left(v_{A}+v_{B}\right) ;(b)$ a comparison between the semi-analytic result and direct simulation data (" $v_{\text {converged" is represented by the }}$ case with $\left.h_{L E} / L_{c}=1 / 10\right)$. The predictions are made at $t a_{\infty} / L_{c}=7.774,7.540,7.461$ and 7.305 for $h_{L E} / L_{c}=0,1 / 30,1 / 15$ and $1 / 10$, respectively, when the vertical velocity reaches its maximum amplitude (see figure 15).

(one grid cell ahead of the peak point) - see (2.2); and, $H$ is the half width of the HV. The bound vortex is located along the WLE, $x=x_{L E}(z)$ that is given by (2.2), and $x_{H}=x_{L E}\left(z_{0}+H\right)$ is the starting $x$-coordinate of the trailing vortices. Here, $x_{H}$ is selected at the position where the largest streamwise vorticity $\left|\omega_{x}\right|$ is obtained.

In (5.3), the cross-sectional circulations $\Gamma_{A}$ and $\Gamma_{B}$ are calculated at each grid point along the HV based on the simulation data by using the Stokes' theorem with a constraint for the $Q$-criterion as

$$
\Gamma=\left.\int_{\mathcal{A}} \omega_{n} \mathrm{~d} \mathcal{A}\right|_{Q>\max (Q) / 100},
$$

where $\mathcal{A}$ is the integration area on a plane that is perpendicular to the WLE curve and accordingly $\omega_{n}$ is the vorticity component normal to the plane. The $Q$-criterion constraint is used to eliminate the circulation produced by the initial prescribed vortex in the current evaluation. Since the prescribed vortex has a significantly lower strength, a value blanking method can be adopted for this purpose. This is achieved by restricting $\mathcal{A}$ to the region where the $Q$-criterion remains above $1 \%$ of the global maximum value. Since the HV structure exists on both the upper and lower sides of the aerofoil, the calculation is repeated for both and added together to obtain the total induced velocity.

The result of the semi-analytic prediction by using (5.3) for the $\mathrm{HV}$-induced vertical velocity in front of the peak is presented in figure 17. The semi-analytic result follows a very similar trend to that from the simulation data albeit not fully matched. It is clearly shown in figure $17 a$ that the net $\mathrm{HV}$-induced vertical velocity decays with increasing $h_{L E}$. This is due to the contributions from the bound and trailing vortices $\left(v_{A}\right.$ and $\left.v_{B}\right)$ cancelling out each other. The mutual cancellation is almost perfect and resulting in a zero sum when $h_{L E} / L_{c}=1 / 15$ or higher, in which case the overall vertical velocity is effectively maintained by the initial vortex approaching (free-field solution). This explains the convergence/saturation of the vertical velocity signal at the peak for high $h_{L E} / L_{c}$, which was questioned in the beginning of the section.

In the meantime, in figure 17, the contribution of the bound vortex $\left(v_{A}\right)$ decreases rapidly and converges towards zero with increasing $h_{L E}$. The rapid decay of $v_{A}$ is considered to be the major cause of the reduction in source strength at the peak discussed earlier (figure 15). This may be explained in three steps. First, between $h_{L E}=0$ (SLE) 

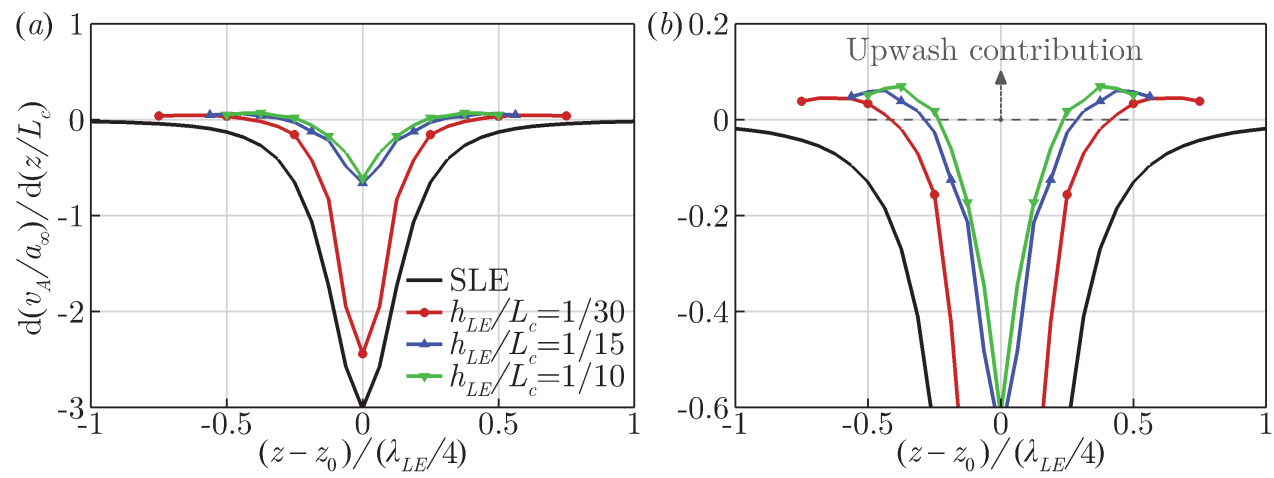

Figure 18. Profiles of $\mathrm{d} v_{A} / \mathrm{d} z$ (normalised by $a_{\infty}$ and $L_{c}$ ) from (5.3): $(a)$ entire and $(b)$ zoomed-up views, showing local piecewise contributions of the bound vortex to the overall induced vertical velocity in front of the peak resulted in figure $17 a$.

and $1 / 30$, there is a large drop in the spanwise vorticity $\left(\omega_{z}\right)$ and the appearance of $\omega_{x}$, which are shown in figure 14. Secondly, between $h_{L E}=1 / 30$ and $1 / 15$, there is a moderate drop in $\omega_{z}$ and a further growth in $\omega_{x}$ leading to a continued reduction of $v_{A}$ up to this point. Lastly, between $h_{L E}=1 / 15$ and $1 / 10, \omega_{x}$ still grows consistently (with very little change in $\omega_{z}$ ); however, this does not reflect efficiently on $v_{A}$ due to the fact that the term $\left[\left(z-z_{0}\right) \mathrm{d} x_{L E} / \mathrm{d} z-\left(x_{L E}-x_{0}\right)\right]$ in (5.3) tends to zero or a very small number (except for the area nearest to the peak where $\omega_{z}$ prevails) since $\mathrm{d} x_{L E} / \mathrm{d} z \sim\left(x_{L E}-x_{0}\right) /\left(z-z_{0}\right)$ as the WLE profile becomes increasingly slender (similar to a long and narrow triangle). These trends can also be found in figure 18 which provides local piecewise contributions of the bound vortex $\left(\mathrm{d} v_{A} / \mathrm{d} z\right.$ as a function of $z$ ) showing the rapid decrease of the bound vortex contribution and its convergence at high $h_{L E} / L_{c}$. The figure also shows a certain amount of upwash generated by the bound vortex indicating the influence of the streamwise vorticity component prevalent around the edges of the bound vortex.

More information can be found from the semi-analytic prediction data. In figure 17 the contribution of the trailing vortices $\left(v_{B}\right)$ seems to gradually decreases with increasing $h_{L E}$ although the streamwise vorticity $\left(\omega_{x}\right)$ grows steadily with $h_{L E}$ as shown in figures 13 and 14. It may be explained by using (5.3) where $v_{B}$ is proportional to $H$ and asymptotically to $\left(x_{H}-x_{0}\right)^{-3}$. For a higher $h_{L E}, H$ decreases and $x_{H}$ increases (as shown in figures 14 and 16), which may result in a reduced $v_{B}$ despite a higher $\omega_{x}$ or $\Gamma_{B}$.

\section{Additional findings and discussions}

This section briefly presents additional findings made with regard to two different aspects based on the current simulation framework: 1) the relationship between the aeroacoustic source strength and the WLE aspect ratio; and, 2) the effect of a threedimensional profile introduced in the impinging vortex on the interaction with the leading-edge geometries.

\subsection{WLE aspect ratio and source strength}

A parametric study is carried out to find a relationship between the aeroacoustic source strength and the WLE aspect ratio $\left(A R=2 h_{L E} / \lambda_{L E}\right)$. Three different values of $A R=0.5,1$ and 1.5 have been studied in $\S 3$ to $\S 5$ with the wavelength fixed at $\lambda_{L E} / L_{c}=2 / 15$. In this section, additional simulations are performed with both $h_{L E}$ and $\lambda_{L E}$ doubled (hence, the aspect ratios remain the same) in order to investigate 

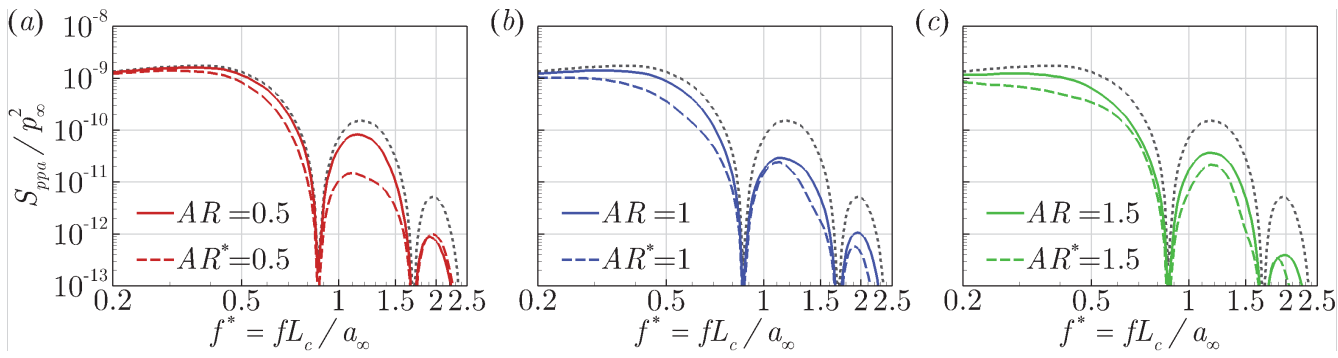

Figure 19. Far-field acoustic pressure PSD profiles, $S_{p p a}\left(f^{*}\right) / p_{\infty}^{2}$, obtained at an observer location, $\boldsymbol{x}_{o}=\left(0,5 L_{c}, 0\right)$, for three different WLE aspect ratios $\left(A R=2 h_{L E} / \lambda_{L E}=0.5,1\right.$ and 1.5). The aspect ratios with "*" indicate that the size of the WLE is doubled $\left(A R^{*}=2 h_{L E}^{*} / \lambda_{L E}^{*}\right.$ where $h_{L E}^{*}=2 h_{L E}$ and $\left.\lambda_{L E}^{*}=2 \lambda_{L E}\right)$. The dotted curves are from the SLE baseline case.

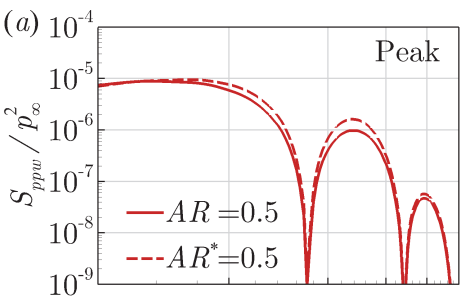

(b)
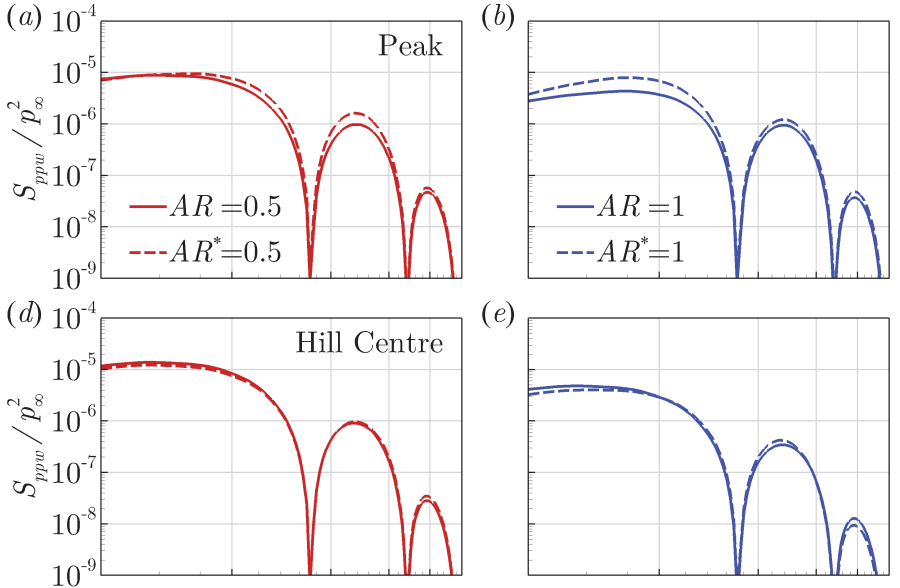

(g) $10^{-4}$

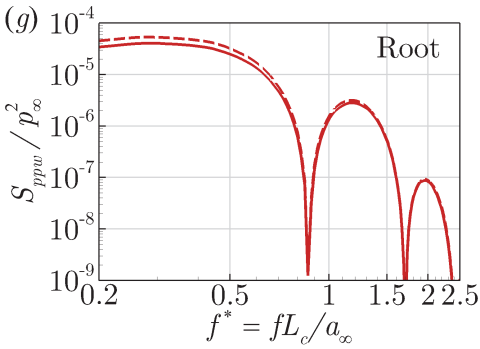

(e)

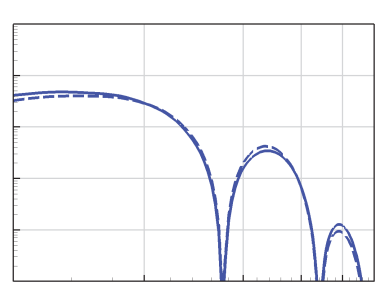

(h)

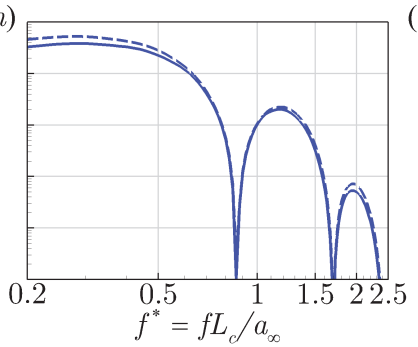

(c)



$(f)$

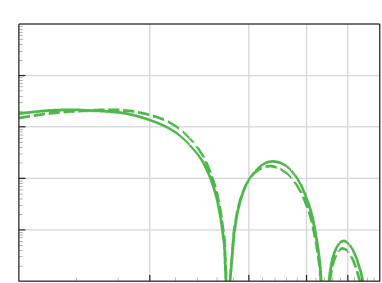

(i)

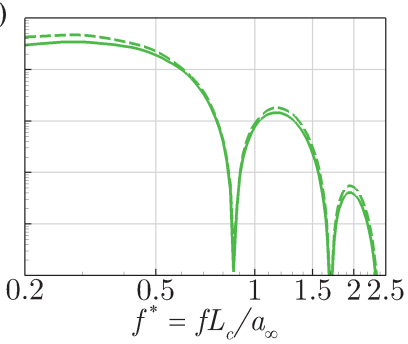

FiguRE 20. Wall pressure PSD profiles, $S_{p p w}\left(f^{*}\right) / p_{\infty}^{2}$, obtained at three different locations on the WLE (peak, hill centre and the root) for three different WLE aspect ratios $\left(A R=2 h_{L E} / \lambda_{L E}=\right.$ $0.5,1$ and 1.5). The aspect ratios with " $*$ " indicate that the size of the WLE is doubled $\left(A R^{*}=2 h_{L E}^{*} / \lambda_{L E}^{*}\right.$ where $h_{L E}^{*}=2 h_{L E}$ and $\left.\lambda_{L E}^{*}=2 \lambda_{L E}\right)$.

the differences in the source characteristics. In terms of the radiated sound pressure level (SPL), it is well known that the SPL is dependent largely on $h_{L E}$ with secondary contributions from $\lambda_{L E}$ (Lau et al. 2013; Narayanan et al. 2015; Chaitanya et al. 2015; Kim et al. 2016). This means that the SPL would be further reduced in the cases of doubled $h_{L E}$ for the same aspect ratio, which is also true in the current study as shown in figure $19\left(A R^{*}=0.5,1\right.$ and 1.5). The further reduction of sound is apparent across the whole frequency range. However, the reduced sound does not necessarily relate to a reduced source strength as far as the current results are concerned - see below. 

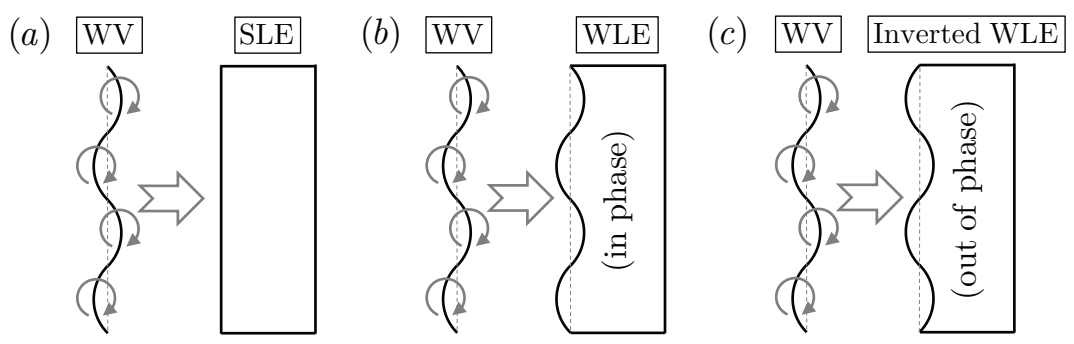

FiguRE 21. Schematics of a wavy vortex (WV) interacting with three different leading-edge geometries: $(a)$ SLE, $(b)$ WLE and $(c)$ the WLE inverted. The centreline of the wavy vortex is undulated along the span in the same fashion applied to the WLE profile - based on (2.2). The inverted WLE (IWLE) has an out-of-phase formation against the prescribed WV.

Figure 20 shows the source strength (wall pressure spectra) at the peak, hill centre and root, obtained from the additional simulations with double sized WLEs, compared with the earlier original cases of the same aspect ratios. It is noticeable that the source strength remains relatively unchanged by doubling the size of the WLE as far as the aspect ratio is maintained. Although slightly large deviations are seen at the peak at some frequencies, a remarkable level of similarities can be found at the root and the hill. The result might suggest that the WLE aspect ratio is a similarity parameter which dictates the source strength and characteristics, despite the radiated sound being influenced mainly by the WLE amplitude. It also suggests that there exist imperative mechanisms contributing to the noise reduction purely associated with the propagation of the sound waves.

One of the propagation effects was indicated by Kim et al. (2016) where it was shown that phase interferences between the source signals make significant contributions to the reduction of the radiated sound at certain frequencies with out-of-phase relationships. The phase interference effect may also be related with the starting frequency of noise reduction, $f_{0} \approx 0.25 U_{\infty} / h_{L E}$, reported by Narayanan et al. (2015). This implies that the low-frequency noise reduction is strongly dependent of the WLE amplitude. However, apart from these, there is little known about the propagation mechanisms particularly with regard to the discrepancies between the source spectra and the radiated sound spectrum as highlighted towards the end of $\S 3.1$. It is envisaged that the similarity in the source characteristics observed in this paper will facilitate the study of the propagation mechanisms in the future if two different WLEs with the same aspect ratio are investigated and compared against each other.

\subsection{The effect of three-dimensional profile in the impinging vortex}

A miscellaneous test has been performed in order to briefly demonstrate the effect of three-dimensionality introduced in the impinging vortex on the interaction with the leading-edge geometries. The schematics of the additional test cases are described in figure 21. The centreline of the impinging vortex no longer remains straight but forms a wavy profile in the same fashion that was applied to the WLEs in the paper. Three additional cases of aerofoil-vortex interaction are considered as depicted in figure 21: (a) WV-SLE, (b) WV-WLE and (c) WV-IWLE, where WV and IWLE stand for wavy vortex and inverted WLE, respectively. The WV and WLE profiles are identical but the IWLE (inverted WLE) has an $180^{\circ}$ phase-shift against the WV. The WV profile is created simply by changing the centre coordinate $\left(x_{0}\right)$ of the vortex used in $(2.5)$ and 

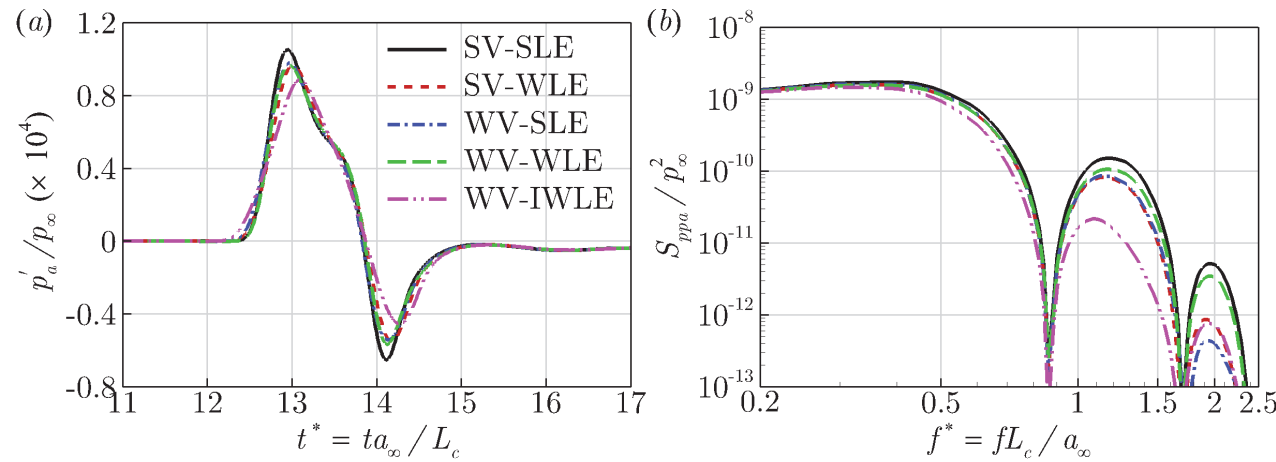

FiguRE 22. Far-field acoustic pressure time signals and the corresponding PSD: $(a) p_{a}^{\prime}\left(t^{*}\right) / p_{\infty}$ and $(b) S_{p p a}\left(f^{*}\right) / p_{\infty}^{2}$ obtained at an observer location, $\boldsymbol{x}_{o}=\left(0,5 L_{c}, 0\right)$, from the additional simulations described in figure 21 . The new results are compared with the earlier cases (SV-SLE and SV-WLE) that used a straight vortex. The WLE and WV profiles are based on $h_{L E} / L_{c}=$ $1 / 30$ and $\lambda_{L E} / L_{c}=2 / 15$.

(2.6) as follows:

$$
x_{0}=-2.5 L_{c}+h_{L E} \sin \left(\frac{2 \pi z}{\lambda_{L E}}\right), \quad z \in\left[-\frac{1}{2} L_{z}, \frac{1}{2} L_{z}\right] .
$$

The result of the additional simulations is presented in figure 22 in comparison with the earlier cases (SV-SLE and SV-WLE) that was based on a straight vortex (SV). It is shown that the WV-WLE case yields an almost identical sound pressure spectrum to that of the SV-SLE case. Also, the WV-SLE and SV-WLE cases are remarkably similar to each other except a marginal difference at high frequencies. In comparison, the WV-IWLE case exhibits a substantial noise reduction across a wide range of frequencies. These results could be explained by looking at the streamwise distance between the impinging vortex and the leading edge. First, the WV-WLE and SV-SLE cases have the same relative distance that is constant along the span. Therefore the impact takes place synchronously (with no time delay) across the span. The WV-SLE and SV-WLE cases also have the same relative distance but varying along the span, which results in an asynchronous interaction over the span, hence a reduced noise level. Finally, the WV-IWLE case is effectively comparable to the SV-WLE case with the WLE amplitude doubled $\left(h_{L E} / L_{c}=1 / 15\right.$ instead of $1 / 30$ ) and therefore resulting in a significantly increased noise reduction (see figure 5).

It is possible to consider more complex cases such as the wavy vortex has a different wavelength, amplitude or phase-shift to those of the wavy leading edge to interact with. Also, the impinging vortex can be finite in span (similar to an individual eddy) as explored in Mathews \& Peake (2015). Studying these increasingly complex cases may enhance the understanding of the noise generation/reduction mechanisms into more details later on.

\section{Concluding remarks}

Detailed understandings of the aeroacoustic source mechanisms of a thin aerofoil with a WLE interacting with a prescribed impinging vortex have been achieved by using high-order accurate aeroacoustics simulations in this paper. The numerical flow visualisation showed that the WLE upon interaction with the impinging vortex created a horseshoe vortex (HV) system stemming from the peak area. The HV-induced vorticity and velocity fields around the WLE provided key information to quantify and understand 
the changes in the wall pressure fluctuation compared to the SLE baseline cases. Two distinctive source behaviours were observed and questioned during the initial analysis of the simulation data. Some scientific explanations to the questions have emerged after investigating into the role of the HV system and the induced velocity field induced by it.

The aeroacoustic source strength at the root remained consistent (similar to that of the SLE counterpart) irrespective of the WLE amplitude $\left(h_{L E}\right)$. This initially seemed incompatible with the growing contribution of the HVs substantially amplifying the vertical velocity perturbation around the root with increasing $h_{L E}$. The current investigation showed that the oblique interaction between the vertical velocity perturbation and the root area could be one of the mechanisms to explain the paradoxical event. From another point of view, it was demonstrated that the area-averaged vertical velocity remained at a constant level irrespective of $h_{L E}$ (indicating the conservation of vertical momentum), which might suggest that the source strength at the root was determined mainly by the overall amount of vertical momentum possessed in the upstream of the root.

Unlike the root, the source strength at the peak was significantly reduced with increasing $h_{L E}$ up to a certain point. The reduction of source strength at the peak was well correlated with the growth in streamwise vorticity and the decay in the spanwise vorticity along the $\mathrm{HV}$ which efficiently attenuated the vertical velocity perturbation. However, the source strength at the peak ceased to decrease further when $h_{L E}$ was sufficiently large. The source convergence/saturation event was explained by using a semi-analytic model of the HV (a curved bound vortex connected with two straight trailing vortices) based on Biot-Savart's law. The semi-analytic model showed that the HV-induced downwash and upwash components eventually led to a complete mutual cancellation as $h_{L E}$ becomes large, and therefore the net vertical velocity in front of the peak converged to that of the free-field solution with no aerofoil in place. It was also found that the highly slender WLE geometry made the HV effectively non-responsive to the source strength at the peak despite the intensified streamwise vorticity components surrounding it.

It appeared that there was a strong similarity rule existing in the source characteristics between two different WLE geometries with the same aspect ratio. The level of similarity viewed from the wall pressure spectra was maintained at all frequencies and all probing points (peak, hill centre and root). This might suggest that the source mechanisms explained in this paper have a certain level of universality applicable to a wide range of WLE geometries. The authors suggest within this hypothesis that it is necessary to investigate the "propagation" mechanisms in detail since there is a significant disagreement between the source power spectra at the wall and the noise reduction spectrum at the far field, as addressed in $§ 3.1$. Also, the exponential growth of noise reduction with frequency - reported repeatedly by Narayanan et al. (2015); Chaitanya et al. (2015); Kim et al. (2016) - is unexplained solely by the source mechanisms.

The current study was focused on the most basic scenario where the impinging vortex was kept two-dimensional and only the leading-edge geometry was changed from $2 \mathrm{D}$ to $3 \mathrm{D}$ such that it was straightforward to identify the differences that the geometric modification made to the flow and sound. It would be necessary afterwards to consider a more complex case where the impinging vortex has a three-dimensional profile as briefly demonstrated in $§ 6.2$. It will aid better understanding the realistic aerofoil-turbulence interaction that has a certain spanwise length scale and coherence. The size of the parametric space to explore will become substantial in that case though. 


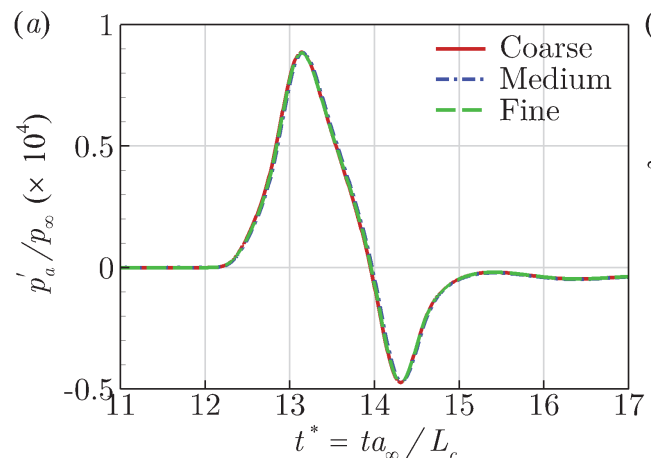

$(c)$
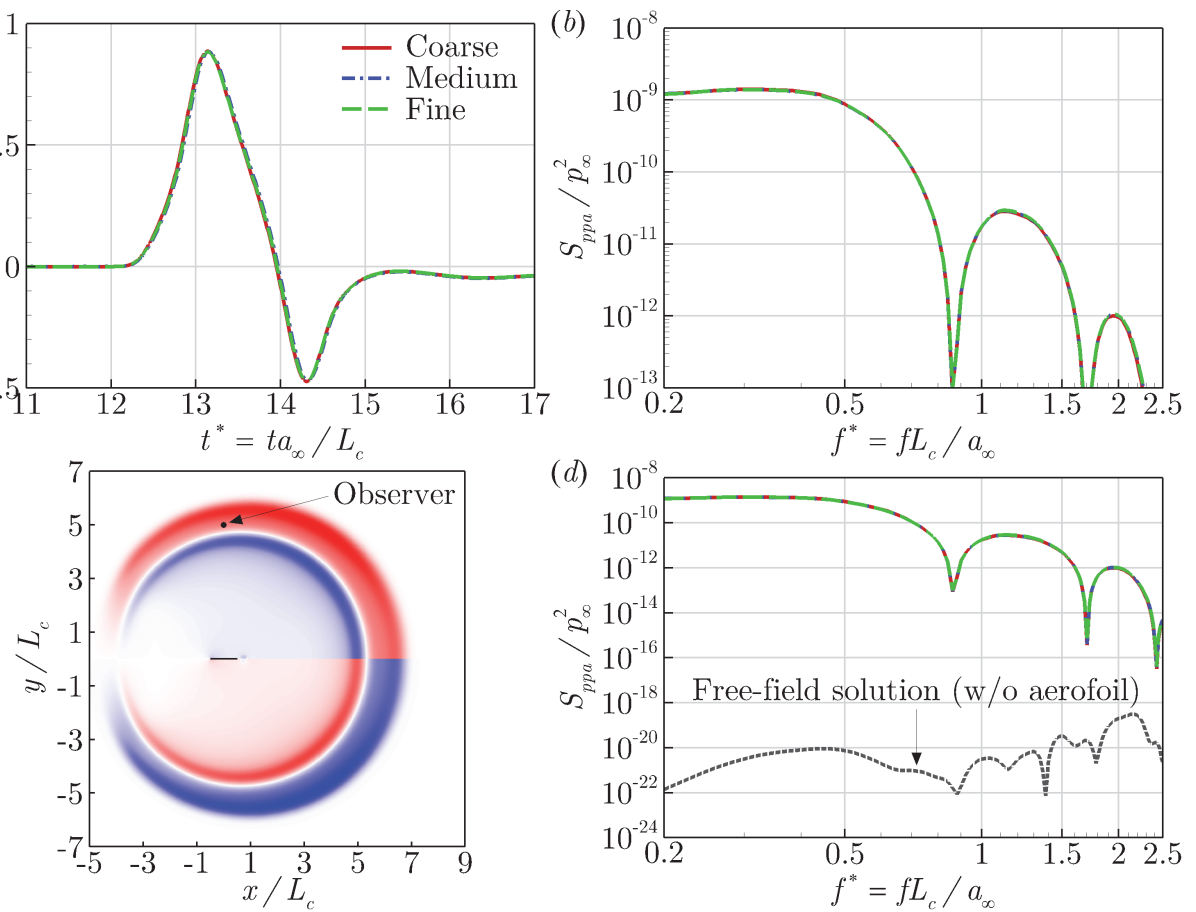

FiguRE 23. A grid convergence test of the current numerical simulation by using three different levels of grid resolution. The far-field acoustic pressure signal $\left(p_{a}^{\prime} / p_{\infty}\right)$ and its PSD $\left(S_{p p a} / p_{\infty}^{2}\right)$ are obtained at an observer point $\boldsymbol{x}_{0}=\left(0,5 L_{c}, 0\right)$. The WLE geometry is based on $h_{L E} / L_{c}=1 / 15$ and $\lambda_{L E} / L_{c}=2 / 15$ (corresponding to figure 5).

\section{Acknowledgement}

The authors gratefully acknowledge the support of EPSRC (Engineering and Physical Sciences Research Council) for the present work under the CDT (Centre for Doctoral Training) grant EP/G03690X/1 and the ICSS (Institute for Complex Systems Simulation) at the University of Southampton. We also acknowledge the high-performance computing facilities and services of the UK National Supercomputer ARCHER via the support of UK Turbulence Consortium (EP/L000261/1) and the local IRIDIS4 at the University of Southampton in the completion of this work.

\section{Appendix A. Validation of computational data}

Figure 23 shows the results of a grid convergence test conducted with a WLE geometry: $h_{L E} / L_{c}=1 / 15$ and $\lambda_{L E} / L_{c}=2 / 15(A R=1)$. Three different levels of grid resolution (coarse, medium and fine) were used. The medium level was used for all simulation data presented in this paper. The coarse/fine levels have $20 \%$ less/more resolution in all directions. The number of grid points and the typical mesh sizes used for each resolution level are listed in table 2. Figures $23 a$ and $23 b$ demonstrates that there is effectively no difference in the results (time signals and the spectra as well) between the three different grids. Figure $23 d$ also shows a comparison to the free-field solution obtained without the aerofoil in place (using the medium grid). The level of background noise (numerical error residual) remained at least 40dB lower than the physical SPL throughout the entire 
frequency range, which represents the quality of the numerical solutions presented in this paper.

\begin{tabular}{cccccc}
\hline Resolution level & $n_{\xi}$ & $n_{\eta}$ & $n_{\zeta}$ & $\Delta x_{\max } / L_{c}$ & $\Delta x_{\min } / L_{c}$ \\
\hline Coarse & 836 & 528 & 52 & 0.012 & 0.0075 \\
Medium & 1036 & 660 & 64 & 0.01 & 0.00625 \\
Fine & 1254 & 792 & 78 & 0.008 & 0.005 \\
\hline
\end{tabular}

TABLE 2. Supplementary information for the grid convergence test shown in figure 23, where $n_{\xi}, n_{\eta}$ and $n_{\zeta}$ denote the number of grid cells in the $\xi$-, $\eta$ - and $\zeta$-directions, respectively - see $(2.3)$ and (2.4).

\section{Appendix B. Sensitivity test for the measure of the obliqueness in interaction}

The following tables show the estimated angles of the oblique interaction $(\phi)$ described in $\S 4$ for various values of the $-v / a_{\infty}$ contour threshold selected from figure $10 c$. The sensitivity test was performed for all three WLE geometries used in the paper $\left(h_{L E} / L_{c}=1 / 30,1 / 15\right.$ and $\left.1 / 10\right)$. The tables show that the choice of the threshold (within the $40 \%$ bandwidth tested) makes an insignificant change to the estimated obliqueness angle with a deviation of up to $4.07 \%$ at maximum as far as the current test cases are concerned.

\begin{tabular}{ccccc}
\hline Threshold selected & $\phi_{1}$ & $\phi_{2}$ & $\phi=\left(\phi_{1}+\phi_{2}\right) / 2$ & Relative difference in $\phi$ \\
\hline $0.00784(-20 \%)$ & $30.18^{\circ}$ & $45.03^{\circ}$ & $37.61^{\circ}$ & $-3.39 \%$ \\
$0.00882(-10 \%)$ & $29.48^{\circ}$ & $46.34^{\circ}$ & $37.91^{\circ}$ & $-2.62 \%$ \\
0.0098 (original) & $30.13^{\circ}$ & $47.72^{\circ}$ & $38.93^{\circ}$ & - \\
$0.01078(+10 \%)$ & $34.43^{\circ}$ & $45.11^{\circ}$ & $39.77^{\circ}$ & $+2.16 \%$ \\
$0.01176(+20 \%)$ & $32.46^{\circ}$ & $45.01^{\circ}$ & $38.74^{\circ}$ & $-0.49 \%$ \\
\hline
\end{tabular}

TABLE 3. The estimation of $\phi$ for various thresholds in the case of $h_{L E} / L_{c}=1 / 30$.

\begin{tabular}{ccccc}
\hline Threshold selected & $\phi_{1}$ & $\phi_{2}$ & $\phi=\left(\phi_{1}+\phi_{2}\right) / 2$ & Relative difference in $\phi$ \\
\hline $0.00784(-20 \%)$ & $42.85^{\circ}$ & $61.42^{\circ}$ & $52.14^{\circ}$ & $-4.07 \%$ \\
$0.00882(-10 \%)$ & $44.75^{\circ}$ & $62.80^{\circ}$ & $53.78^{\circ}$ & $-1.05 \%$ \\
0.0098 (original) & $45.37^{\circ}$ & $63.33^{\circ}$ & $54.35^{\circ}$ & - \\
$0.01078(+10 \%)$ & $44.34^{\circ}$ & $61.45^{\circ}$ & $52.90^{\circ}$ & $-2.67 \%$ \\
$0.01176(+20 \%)$ & $43.42^{\circ}$ & $61.10^{\circ}$ & $52.26^{\circ}$ & $-3.85 \%$ \\
\hline
\end{tabular}

TABLE 4 . The estimation of $\phi$ for various thresholds in the case of $h_{L E} / L_{c}=1 / 15$. 


\begin{tabular}{ccccc}
\hline Threshold selected & $\phi_{1}$ & $\phi_{2}$ & $\phi=\left(\phi_{1}+\phi_{2}\right) / 2$ & Relative difference in $\phi$ \\
\hline $0.00784(-20 \%)$ & $48.97^{\circ}$ & $69.55^{\circ}$ & $59.26^{\circ}$ & $-1.66 \%$ \\
$0.00882(-10 \%)$ & $47.97^{\circ}$ & $69.23^{\circ}$ & $58.60^{\circ}$ & $-2.75 \%$ \\
0.0098 (original) & $50.76^{\circ}$ & $69.76^{\circ}$ & $60.26^{\circ}$ & - \\
$0.01078(+10 \%)$ & $51.08^{\circ}$ & $67.97^{\circ}$ & $59.53^{\circ}$ & $-1.21 \%$ \\
$0.01176(+20 \%)$ & $51.00^{\circ}$ & $67.45^{\circ}$ & $59.23^{\circ}$ & $-1.71 \%$ \\
\hline
\end{tabular}

TABLE 5. The estimation of $\phi$ for various thresholds in the case of $h_{L E} / L_{c}=1 / 10$.

Amiet, R. K. 1975 Acoustic radiation from an airfoil in a turbulent stream. J. Sound Vib. 41, 407-420.

Atassi, H. M., Subramaniam, S. \& Scott, J. R. 1990 Acoustic radiation from lifting airfoils in compressible subsonic flow. AIAA 90-3911, Washington, D.C., U.S.A.

Ayton, L. J. \& Peake, N. 2013 On high-frequency noise scattering by aerofoils in flow. $J$. Fluid Mech. 734, 144-182.

Ayton, L. J. \& Peake, N. 2015 On high-frequency sound generated by gust-aerofoil interaction in shear flow. J. Fluid Mech. 766, 297-325.

Chaitanya, P., Narayanan, S., Joseph, P., Vanderwel, C., Turner, J., Kim, J. W. \& Gantapathisubramani, B. 2015 Broadband noise reductions through leading edge serrations on realistic aerofoils. In 21st AIAA/CEAS Aeroacoustics Conference.

Christophe, J., Anthoine, J. \& Rambaud, P. 2008 Numerical issues in the application of an Amiet model for spanwise-varying incoming turbulence. In 14th AIAA/CEAS Aeroacoustics Conference. AIAA Paper 2008-1600.

Christophe, J., Anthoine, J., Rambaud, P., Schram, C., Mathey, F. \& Moreau, S. 2007 Prediction of incoming turbulent noise using a combined numerical/semi-empirical method and experimental validation. In 13th AIAA/CEAS Aeroacoustics Conference. AIAA Paper 2007-3468.

Clair, V., Polacsek, C., Garrec, T. L., Reboul, G., Gruber, M. \& Joseph, P. 2013 Experimental and numerical investigation of turbulence-airfoil noise reduction using wavy edges. AIAA J. 51, 2695-2713.

Deniau, H., , Boussuge, J. F., Polacsek, C. \& Moreau, S. 2011 Affordable compressible LES of airfoil-turbulence interaction in a free jet. In 17th AIAA/CEAS Aeroacoustics Conference. AIAA Paper 2011-2707.

Devenport, W. J., Staubs, J. K. \& GlegG, S. A. L. 2010 Sound radiation from real airfoils in turbulence. J. Sound Vib. 329, 3470-3483.

Evers, I. \& Peake, N. 2000 Noise generation by high-frequency gusts interacting with an airfoil in transonic flow. J. Fluid Mech. 411, 91-130.

Fish, F. E., Howle, L. E. \& Murray, M. M. 2008 Hydrodynamic flow control in marine mammals. Integr. Comp. Biol. 48, 788-800.

Gill, J., Zhang, X. \& Joseph, P. 2013 Symmetric airfoil geometry effects on leading edge noise. J. Acoust. Soc. Am. 134, 2669-2680.

Gill, J., Zhang, X. \& Joseph, P. F. 2015 Single velocity-component modeling of leading edge turbulence interaction noise. J. Acoust. Soc. Am. 137 (6), 3209-3220.

Goldstein, M. E. 1978 Unsteady vortical and entropic distortions of potential flows around arbitrary obstacles. J. Fluid Mech. 89, 433-468.

Hansen, K., Kelso, R. \& Doolan, C. 2012 Reduction of flow induced airfoil tonal noise using leading edge sinusoidal modifications. Acoust. Australia 40 (3), 172-177.

Hansen, K. L., Kelso, R. M. \& Dally, B. D. 2011 Performance variations of leading-edge tubercles for distinct airfoil profiles. AIAA J. 49, 185-194.

Hansen, K. L., Rostamzadeh, N., Kelso, R. M. \& Dally, B. B. 2016 Evolution of the streamwise vortices generated between leading edge tubercles. J. Fluid Mech. 788, 730766.

Johari, H., Henoch, C., Custodio, D. \& Levshin, L. 2007 Effects of leading-edge protuberances on airfoil performance. AIAA J. 45, 2634-2642. 
KIM, J. W. 2007 Optimised boundary compact finite difference schemes for computational aeroacoustics. J. Comput. Phys. 225, 995-1019.

KIM, J. W. 2010 High-order compact filters with variable cut-off wavenumber and stable boundary treatment. Comput. Fluids 39, 1168-1182.

KIM, J. W. 2013 Quasi-disjoint pentadiagonal matrix systems for the parallelization of compact finite-difference schemes and filters. J. Comput. Phys. 241, 168-194.

Kim, J. W. \& HAeri, S. 2015 An advanced synthetic eddy method for the computation of aerofoil-turbulence interaction noise. J. Comput. Phys. 287, 1-17.

Kim, J. W., Haeri, S. \& Joseph, P. 2016 On the reduction of aerofoil-turbulence interaction noise associated with wavy leading edges. J. Fluid Mech. 792, 526-552.

Kim, J. W., LaU, A. S. H. \& Sandham, N. D. 2010 Proposed boundary conditions for gustairfoil interaction noise. AIAA J. 48 (11), 2705-2710.

KIM, J. W. \& LEE, D. J. 2000 Generalized characteristic boundary conditions for computational aeroacoustics. AIAA J. 38 (11), 2040-2049.

KIM, J. W. \& LEE, D. J. 2004 Generalized characteristic boundary conditions for computational aeroacoustics, part 2. AIAA J. 42 (1), 47-55.

Kim, J. W. \& Morris, P. J. 2002 Computation of subsonic inviscid flow past a cone using high-order schemes. AIAA J. 40 (10), 1961-1968.

LAu, A. S. H., HAERI, S. \& Kim, J. W. 2013 The effect of wavy leading edges on aerofoil-gust interaction noise. J. Sound Vib. 25, 6234-6253.

LockArd, D. \& Morris, P. 1998 Radiated noise from airfoils in realistic mean flows. AIAA J. 36, 907-914.

Mathews, J. \& PEAKe, N. 2015 Noise generation by turbulence interacting with an aerofoil with a serrated leading edge. In 21st AIAA/CEAS Aeroacoustics Conference. AIAA Paper 2015-2204.

Migliore, P. \& Oerlemans, S. 2004 Wind tunnel aeroacoustic tests of six airfoils for use on small wind turbines. J. Sol. Energy Eng. 126, 974-985.

Miklosovic, D. S., Murray, M. M., Howle, L. E. \& Fish, F. E. 2004 Leading-edge tubercles delay stall on humpback whale flippers. Phys. Fluids 16, 39-42.

Moreau, S., Roger, M. \& Jurdic, V. 2005 Effect of angle of attack and airfoil shape on turbulence-interaction noise. In 11th AIAA/CEAS Aeroacoustics Conference. AIAA Paper 2005-2973.

Myers, M. R. \& Kerschen, E. J. 1995 Influence of incidence angle on sound generation by airfoils interacting with high-frequency gusts. J. Fluid Mech. 292, 271-304.

Myers, M. R. \& Kerschen, E. J. 1997 Influence of camber on sound generation by airfoils interacting with high-frequency gusts. J. Fluid Mech. 353, 221-259.

Narayanan, S., Chaitanya, P., Haeri, S., Joseph, P., Kim, J. W. \& Polacsek, C. 2015 Airfoil noise reductions through leading edge serrations. Phys. Fluids 27, 025109.

Paterson, R. W. \& Amiet, R. K. 1976 Acoustic radiation and surface pressure characteristics of an airfoil due to incident turbulence. NASA CR-2733.

Rockwell, D. 1998 Vortex-body interactions. Annu. Rev. Fluid Mech. 30, 199-229.

Roger, M. \& Moreau, S. 2010 Extensions and limitations of analytical airfoil broadband noise models. Int. J. Aeroacoust. 9, 273-305.

Tsuji, Y., Fransson, J. H. M., Alfredsson, P. H. \& Johansson, A. V. 2007 Pressure statistics and their scaling in high-reynolds-number turbulent boundary layers. J. Fluid Mech. 585, 1-40. 